\title{
The Finite Larmor Radius Approximation
}

\author{
Emmanuel Frénod, Eric Sonnendrücker
}

\section{$\mathbf{N}^{\circ} 3847$}

Décembre 1999

THÈME 4

\section{apport}

de recherche 



\title{
RINRIA
}

\section{The Finite Larmor Radius Approximation}

\author{
Emmanuel Frénod*, Eric Sonnendrücker ${ }^{\dagger}$ \\ Thème 4 - Simulation et optimisation \\ de systèmes complexes \\ Projet Numath \\ Rapport de recherche $\mathrm{n}^{\circ} 3847$ - Décembre 1999 - 23 pages
}

\begin{abstract}
The presence of a large external magnetic field in a plasma introduces an additional time-scale which is very constraining for the numerical simulation. Hence it is very useful to introduce averaged models which remove this time-scale. However, depending on other parameters of the plasma different starting models for the asymptotic analysis may be considered. We introduce here a generic framework for our analysis which fits many of the possible regimes and apply it in particular to justify the Finite Larmor Radius approximation both in the linear case and in the non linear case in the plane transverse to the magnetic field.
\end{abstract}

Key-words: Vlasov-Poisson equations, kinetic equations, homogenization, multiple time scales, two scale convergence

(Résumé : tsvp)

* Laboratoire de Mathématiques et Applications des Mathématiques, Université de Bretagne Sud, Vannes

$\dagger$ CNRS, Institut Elie Cartan, Université Henri Poincaré, Nancy \& Institut de Recherche en Mathématiques Avancées, Université Louis Pasteur, Strasbourg

Unité de recherche INRIA Lorraine

Technopôle de Nancy-Brabois, Campus scientifique, 615 rue de Jardin Botanique, BP 101, 54600 VILLERS LÈS NANCY (France)

Téléphone : 0383593030 - International : +33 3383593030

Télécopie : 0383278319 - International : +33 383278319

Antenne de Metz, technopôle de Metz 2000, 4 rue Marconi, 55070 METZ

Téléphone : 0387203500 - Internatională: +33 387203500

Télécopie : 0387763977 - International : +33 387763977 


\section{L'approximation Rayon de Larmor Fini}

Résumé : La présence d'un fort champ magnétique extérieur dans un plasma introduit une échelle de temps supplémentaire qui est très contraignante pour la simulation numérique. Pour cette raison, il peut être très utile d'introduire des modèles approchés qui éliminent cette échelle de temps. En fonction des autres grandeurs caractéristiques du plasma considéré, différentes asymptotiques peuvent être considérées. Nous indroduisons un cadre générique pour notre analyse, et nous appliquons ensuite les résultats obtenus aux différents régimes ce qui nous permet en particulier d'obtenir l'approximation "Rayon de Larmor Fini" aussi bien dans le cas linéaire que dans le cas non linéaire dans le plan perpendiculaire au champ magnétique.

Mots-clé : Equations de Vlasov-Poisson, équations cinétiques, homogénéisation, échelles de temps multiples, convergence à deux échelles 


\section{Introduction}

The main goal of this paper is the investigation of an asymptotic regime taking place in the description of the behavior of charged particles under the action of a strong external magnetic field and called: "The Finite Larmor Radius Approximation". This approximation has a natural field of application in tokamak physics.

This work was announced in E. Frénod \& E. Sonnendrücker [8] and follows E. Frénod \& E. Sonnendrücker $[9,7]$ where we exhibited global asymptotic behavior of plasmas. Those global behaviors have also been mathematically put in light by F. Golse \& L. Saint Raymond [11, 10] and E. Grenier [13]. The context of "The Finite Larmor Radius Approximation" is more local. Its object is to describe the behavior of the considered plama's particles when the observation length scale is comparable with their Larmor radius.

We choose to lead our study in the framework of the Vlasov equation which writes, in this context

$$
\begin{aligned}
& \frac{\partial f^{\varepsilon}}{\partial t}+\mathbf{v}_{\|} \cdot \nabla_{x} f^{\varepsilon}+\frac{1}{\varepsilon} \mathbf{v}_{\perp} \cdot \nabla_{x} f^{\varepsilon}+\left(\mathbf{E}+\frac{1}{\varepsilon} \mathbf{v} \times \mathcal{M}\right) \cdot \nabla_{v} f^{\varepsilon}=0, \\
& f_{\mid t=0}^{\varepsilon}=f_{0},
\end{aligned}
$$

where $\varepsilon$ is a small parameter which will tend to 0 . In (1.1) the distribution function $f^{\varepsilon} \equiv f^{\varepsilon}(t, \mathbf{x}, \mathbf{v})$; $t \in[0, T)$ for some $T<\infty$ is the time, $\mathbf{x}=\left(x_{1}, x_{2}, x_{3}\right) \in \mathbb{R}_{x}^{3}$ is the position and $\mathbf{v}=\left(v_{1}, v_{2}, v_{3}\right) \in \mathbb{R}_{v}^{3}$ is the velocity. We denote $\mathcal{O}=\mathbb{R}_{x}^{3} \times \mathbb{R}_{v}^{3}, \Omega=[0, T) \times \mathbb{R}_{x}^{3}$ and $\mathcal{Q}=[0, T) \times \mathcal{O}$. The magnetic field $\mathcal{M}$ is supposed to be $\mathbf{e}_{1}$, the first vector of the frame $\left(\mathbf{e}_{1}, \mathbf{e}_{2}, \mathbf{e}_{3}\right)$ of $\mathbb{R}^{3}$. For any vector $\mathbf{v} \in \mathbb{R}^{3}, \mathbf{v}_{\|}$stands for $\mathbf{v}_{\|}=(\mathbf{v} \cdot \mathcal{M}) \mathcal{M}=v_{1} \mathbf{e}_{1}$ and $\mathbf{v}_{\perp}$ for $\mathbf{v}_{\perp}=\mathbf{v}-\mathbf{v}_{\|}=v_{2} \mathbf{e}_{2}+v_{3} \mathbf{e}_{3}$. The electric field $\mathbf{E} \equiv \mathbf{E}(t, \mathbf{x})$ is external and non oscillating.

In order to make the process $\varepsilon \rightarrow 0$ in (1.1), we assume

$$
f_{0} \geq 0, f_{0} \in L^{1} \cap L^{2}(\mathcal{O}),
$$

and for $\mathbf{E}$, we assume

$$
\mathbf{E} \in \mathcal{C}^{1}(\Omega)
$$

Then we have the following Theorem.

THEOREM 1.1 Under assumptions (1.2) and (1.3), for each $\varepsilon>0$, there exists a unique solution $f^{\varepsilon}$ of (1.1) in $L^{\infty}\left(0, T, L^{1} \cap L^{2}(\mathcal{O})\right)$. As $\varepsilon \rightarrow 0$,

$$
f^{\varepsilon} \rightarrow f \text { in } L^{\infty}\left(0, T, L^{2}(\mathcal{O})\right) \text { weak }-*,
$$

where $f$ is the unique solution of

$$
\begin{aligned}
& \frac{\partial f}{\partial t}+\mathbf{v}_{\|} \cdot \nabla_{x} f+\frac{1}{2 \pi}\left(\int_{0}^{2 \pi} \mathcal{R}(-\tau) \mathbf{E}(t, \mathbf{x}+\mathcal{R}(\tau) \mathbf{v}) d \tau\right) \cdot \nabla_{x} f \\
& \quad+\frac{1}{2 \pi}\left(\int_{0}^{2 \pi} R(-\tau) \mathbf{E}(t, \mathbf{x}+\mathcal{R}(\tau) \mathbf{v}) d \tau\right) \cdot \nabla_{v} f=0 \\
& f_{\mid t=0}=\frac{1}{2 \pi} \int_{0}^{2 \pi} f_{0}(\mathbf{x}+\mathcal{R}(-\tau) \mathbf{v}, R(-\tau) \mathbf{v}) d \tau
\end{aligned}
$$

where the matrices $R$ and $\mathcal{R}$ are given by

$$
R(\tau)=\left(\begin{array}{ccc}
1 & 0 & 0 \\
0 & \cos \tau & \sin \tau \\
0 & -\sin \tau & \cos \tau
\end{array}\right), \mathcal{R}(\tau)=\left(\begin{array}{ccc}
0 & 0 & 0 \\
0 & \sin \tau & 1-\cos \tau \\
0 & \cos \tau-1 & \sin \tau
\end{array}\right)
$$

$\mathrm{RR} \mathrm{n}^{\circ} 3847$ 
The way to prove this Theorem uses the 2-scale convergence that says:

THEOREM 1.2 (N'Guetseng [17] and Allaire [2]) If a sequence $f^{\varepsilon}$ is bounded in $L^{\infty}(0, T ; W)$, for a Banach spaces $W$ being the dual of a separable space and being compactly embedded in $\mathcal{D}^{\prime}(\mathcal{O})$, then for every period $\theta$, there exists a $\theta$-periodic profile $F_{\theta}(t, \tau, \mathbf{x}, \mathbf{v}) \in L^{\infty}\left(0, T ; L_{\theta}^{\infty}\left(\mathbb{R}_{\tau} ; W\right)\right)$ such that for all $\psi_{\theta}(t, \tau, \mathbf{x}, \mathbf{v})$ regular, with compact support with respect to $(t, \mathbf{x}, \mathbf{v})$ and $\theta$-periodic with respect to $\tau$ we have, up to a subsequence,

$$
\int_{\mathcal{Q}} f^{\varepsilon} \psi_{\theta}^{\varepsilon} d t d \mathbf{x} d \mathbf{v} \rightarrow \int_{\mathcal{Q}} \int_{0}^{\theta} F_{\theta} \psi_{\theta} d \tau d t d \mathbf{x} d \mathbf{v}
$$

Above, $L_{\theta}^{\infty}\left(\mathbb{R}_{\tau}\right)$ stands for the space of functions being $L^{\infty}(\mathbb{R})$ and being $\theta$-periodic and $\psi_{\theta}^{\varepsilon} \equiv$ $\psi_{\theta}\left(t, \frac{t}{\varepsilon}, \mathbf{x}, \mathbf{v}\right)$.

The profile $F_{\theta}$ is called the $\theta$-periodic two scale limit of $f^{\varepsilon}$ and the link between $F_{\theta}$ and the weak-* limit $f$ of $\left(f^{\varepsilon}\right)$ is given by

$$
\int_{0}^{\theta} F_{\theta}(t, \tau, \mathbf{x}, \mathbf{v}) d \tau=f(t, \mathbf{x}, \mathbf{v}) .
$$

Moreover, if a sequence $\left(g^{\varepsilon}\right)$ strongly converges to $g$ in a second Banach space $W^{\prime}$ (with the same assumption for $W^{\prime}$ as for $W$ ), such that the product makes sense in a third Banach space $W^{\prime \prime}$, then,

$$
f^{\varepsilon} g^{\varepsilon} \text { 2-scale converges to } F_{\theta} g \in L^{\infty}\left(0, T ; L_{\theta}^{\infty}\left(\mathbb{R}_{\tau} ; W^{\prime \prime}\right)\right) \text {. }
$$

The proof of Theorem 1.1 consist in finding a constraint equation for the two scale limit $F$ of $f^{\varepsilon}$, using a weak formulation with oscillating test function of (1.1). This constraint imposes a form to $F$. Then using oscillating test functions satisfying the constraint equation in the previously evoqued weak formulation gives the equation satisfied by $F$. Integrating this last equation yields finally (1.5).

As the Proof of Theorem 1.1 in this paper and of Theorems 1.1 and 3.2 of E. Frénod \& E. Sonnendrücker [9] are very close, we develop here a generic framework inside which all those proofs may be included. This generic framework consists in considering a conservation law linearly perturbed:

$$
\begin{aligned}
& \frac{\partial u^{\varepsilon}}{\partial t}+\mathbf{A} \cdot \nabla_{x} u^{\varepsilon}+\frac{1}{\varepsilon} \mathbf{L} \cdot \nabla_{x} u^{\varepsilon}=0 \\
& u_{t=0}^{\varepsilon}=u_{0} .
\end{aligned}
$$

In this system, $u^{\varepsilon} \equiv u^{\varepsilon}(t, \mathbf{x}), t \in[0, T)$ for some $T<\infty$ and $\mathbf{x} \in \mathbb{R}^{n}=\mathcal{O}$. We denote $\mathcal{Q}=[0, T) \times \mathcal{O}$, and we assume $\mathbf{A} \equiv \mathbf{A}(t, \mathbf{x}) \in L^{\infty}\left(0, T ; L_{l o c}^{2}(\mathcal{O})\right)$, with $\nabla_{x} \cdot \mathbf{A}=0$ and $\mathbf{L} \equiv M \mathbf{x}+N$ where $M$ is a real $n \times n$ matrix with constant entries, satisfying $\operatorname{tr} M=0$ and where $N \in \operatorname{Im} M$. We moreover assume that $e^{\tau M}$ is $\theta$-periodic for a given $\theta \in \mathbb{R}$. The generic Theorem writes:

THEOREM 1.3 Under the assumptions above, if moreover the sequence $\left(u^{\varepsilon}\right)$ of solution of (1.10) satisfies

$$
\left\|u^{\varepsilon}\right\|_{L^{\infty}\left(0, T ; L^{2}(\mathcal{O})\right)} \leq C
$$

for some constants $C$ independent on $\varepsilon$, then extracting a subsequence,

$u^{\varepsilon}$ two scale converges to a $\theta$ - periodic profile $U \in L^{\infty}\left(0, T ; L^{\infty}\left(\mathbb{R}_{\tau} ; L_{\theta}^{2}(\mathcal{O})\right)\right)$, 
and

$$
u^{\varepsilon} \rightarrow u \text { in } L^{\infty}\left(0, T ; L^{2}(\mathcal{O})\right) \text { weak }-* .
$$

We have,

$$
U(t, \tau, \mathbf{x})=U_{0}\left(e^{-\tau M}(\mathbf{x}-\bar{N})+\bar{N}\right),
$$

where $\bar{N}$ is such that $-M \bar{N}=N$ and where $U_{0} \equiv U_{0}(t, \mathbf{y})$ is solution of

$$
\begin{aligned}
& \frac{\partial U_{0}}{\partial t}+\frac{1}{\theta} \int_{0}^{\theta} e^{-\sigma M} \mathbf{A}\left(t, e^{\sigma M}(\mathbf{y}-\bar{N})+\bar{N}\right) d \sigma \cdot \nabla_{y} U_{0}=0, \\
& U_{0 \mid t=0}=\frac{1}{\theta} u_{0} .
\end{aligned}
$$

Moreover, $u$ is solution of

$$
\begin{aligned}
& \frac{\partial u}{\partial t}+\frac{1}{\theta} \int_{0}^{\theta} e^{-\sigma M} \mathbf{A}\left(t, e^{\sigma M}(\mathbf{x}-\bar{N})+\bar{N}\right) d \sigma \cdot \nabla_{x} u=0 \\
& u_{\mid t=0}(\mathbf{x})=\frac{1}{\theta} \int_{0}^{\theta} u_{0}\left(e^{-\sigma M}(\mathbf{x}-\bar{N})+\bar{N}\right) d \sigma
\end{aligned}
$$

When restricting to the plane perpendicular to $\mathcal{M}$, we may extend the previous result to the Vlasov-Poisson system.

We suppose now that that $f^{\varepsilon}$ does not depend on $x_{1}$ and $v_{1}$, and we use the following notations: $t \in[0, T), T<\infty$ still denotes the time, the position- and velocity-variables become $\mathbf{x}=\left(x_{2}, x_{3}\right) \in$ $\mathbb{R}_{x}^{2}$ and $\mathbf{v}=\left(v_{2}, v_{3}\right) \in \mathbb{R}_{v}^{2}$. We set $\mathcal{O}=\mathbb{R}_{x}^{2} \times \mathbb{R}_{v}^{2}, \Omega=[0, T) \times \mathbb{R}_{x}^{2}$ and $\mathcal{Q}=[0, T) \times \mathcal{O}$. The electric field $\mathbf{E}^{\varepsilon} \equiv \mathbf{E}^{\varepsilon}(t, \mathbf{x})$ standing in the Vlasov equation is now given by the Poisson equation, and then the system we work with writes

$$
\begin{aligned}
& \frac{\partial f^{\varepsilon}}{\partial t}+\frac{1}{\varepsilon} \mathbf{v} \cdot \nabla_{x} f^{\varepsilon}+\left(\mathbf{E}^{\varepsilon}+\frac{1}{\varepsilon} \mathbf{v} \times \mathcal{M}\right) \cdot \nabla_{v} f^{\varepsilon}=0, \\
& f_{\mid t=0}^{\varepsilon}=f_{0}, \\
& \mathbf{E}^{\varepsilon}=-\nabla \phi^{\varepsilon}, \quad-\Delta \phi^{\varepsilon}=\rho^{\varepsilon}, \\
& \rho^{\varepsilon}=\int_{\mathbb{R}_{v}^{2}} f^{\varepsilon} d \mathbf{v} .
\end{aligned}
$$

As the equation (1.17-a) is bidimensional, we precise the sense to give to $\mathbf{v} \times \mathcal{M}$ :

$$
\mathbf{v} \times \mathcal{M}=\left(\begin{array}{c}
v_{3} \\
-v_{2}
\end{array}\right) .
$$

We assume

$$
f_{0} \geq 0, \quad f_{0} \in L^{1} \cap L^{p}(\mathcal{O}), \quad 0<\int_{\mathcal{O}} f_{0}\left(1+|\mathbf{v}|^{2}\right) d \mathbf{v}<+\infty,
$$

for some $p \geq 2$, and we have the following Theorem

$\mathrm{RR} \mathrm{n}^{\circ} 3847$ 
THEOREM 1.4 Under assumption (1.19), for each $\varepsilon$, there exists a solution $\left(f^{\varepsilon}, \mathbf{E}^{\varepsilon}\right)$ of (1.17) in $L^{\infty}\left(0, T ; L^{1} \cap L^{p}(\mathcal{O})\right) \times L^{\infty}\left(0, T ; W^{1, \frac{3}{2}}\left(\mathbb{R}_{x}^{2}\right)\right.$ for any $T \in \mathbb{R}^{+}$. Moreover this solution is bounded in $L^{\infty}\left(0, T ; L^{1} \cap L^{p}(\mathcal{O})\right) \times L^{\infty}\left(0, T ; W^{1, \frac{3}{2}}\left(\mathbb{R}_{x}^{2}\right)\right)$ independently on $\varepsilon$.

If we consider a sequence $\left(f^{\varepsilon}, \mathbf{E}^{\varepsilon}\right)$ of such solutions, extracting a subsequence, we have

$$
\begin{aligned}
& f^{\varepsilon} \text { 2-scale converges to } F \in L^{\infty}\left(0, T ; L_{2 \pi}^{\infty}\left(\mathbb{R}_{\tau} ; L^{p}(\mathcal{O})\right)\right), \\
& \mathbf{E}^{\varepsilon} \text { 2-scale converges to } \mathcal{E} \in L^{\infty}\left(0, T ; L_{2 \pi}^{\infty}\left(\mathbb{R}_{\tau} ; W^{1, \frac{3}{2}}\left(\mathbb{R}_{x}^{2}\right)\right)\right),
\end{aligned}
$$

where $F \equiv F(t, \tau, \mathbf{x}, \mathbf{v})$ and $\mathcal{E} \equiv \mathcal{E}(t, \tau, \mathbf{x})$.

Moreover, there exists a function $G \equiv G(t, \mathbf{y}, \mathbf{u}) \in L^{\infty}\left(0, T ; L^{1} \cap L^{p}\left(\mathcal{O}^{\prime}\right)\right)$ such that

$$
F(t, \tau, \mathbf{x}, \mathbf{v})=G(t, \mathbf{x}+\mathcal{R}(-\tau) \mathbf{v}, R(-\tau) \mathbf{v}),
$$

and $(G, \mathcal{E})$ is solution of

$$
\begin{aligned}
& \frac{\partial G}{\partial t}+\frac{1}{2 \pi}\left(\int_{0}^{2 \pi} \mathcal{R}(-\tau) \mathcal{E}(t, \tau, \mathbf{y}+\mathcal{R}(\tau) \mathbf{u}) d \tau\right) \cdot \nabla_{y} G \\
& +\frac{1}{2 \pi}\left(\int_{0}^{2 \pi} R(-\tau) \mathcal{E}(t, \tau, \mathbf{x}+\mathcal{R}(\tau) \mathbf{u}) d \tau\right) \cdot \nabla_{u} G=0, \\
& G_{\mid t=0}=\frac{1}{2 \pi} f_{0} \\
& \mathcal{E} \equiv \mathcal{E}(t, \tau, \mathbf{x}), \text { with } \mathcal{E}=-\nabla \Phi,-\Delta \Phi=\int_{0}^{2 \pi} G(t, \mathbf{x}+\mathcal{R}(-\tau) \mathbf{v}, R(-\tau) \mathbf{v}) d \mathbf{v}
\end{aligned}
$$

with $R$ and $\mathcal{R}$ given by

$$
R(\tau)=\left(\begin{array}{cc}
\cos \tau & \sin \tau \\
-\sin \tau & \cos \tau
\end{array}\right), \mathcal{R}(\tau)=\left(\begin{array}{cc}
\sin \tau & 1-\cos \tau \\
\cos \tau-1 & \sin \tau
\end{array}\right)
$$

In order to prove this Theorem, we modify the generic framework previously introduced. We consider here

$$
\begin{aligned}
& \frac{\partial u^{\varepsilon}}{\partial t}+\mathbf{A}^{\varepsilon} \cdot \nabla_{x} u^{\varepsilon}+\frac{1}{\varepsilon} \mathbf{L} \cdot \nabla_{x} u^{\varepsilon}=0, \\
& u_{t=0}^{\varepsilon}=u_{0} .
\end{aligned}
$$

where the notations are similar as for equation (1.10): $u^{\varepsilon} \equiv u^{\varepsilon}(t, \mathbf{x}), t \in[0, T), T<\infty ; \mathbf{x} \in \mathbb{R}^{n}=\mathcal{O}$, $\mathcal{Q}=[0, T) \times \mathcal{O}$. We suppose, as previously, that $\mathbf{L} \equiv M \mathbf{x}+N$, where $M$ is a constant entry matrix satisfying $\operatorname{tr} M=0$ and $e^{\tau M}$ is $\theta$-periodic and where $N \in \operatorname{Im} M$. The assumption we make on $\mathbf{A}^{\varepsilon}$ are the following: we suppose that, for all $\varepsilon>0, \nabla_{x} \cdot \mathbf{A}^{\varepsilon}=0$ and that, for some $q>1$,

$$
\mathbf{A}^{\varepsilon} \text { 2-scale converges to } \mathcal{A} \in L^{\infty}\left(0, T ; L_{\theta}^{\infty}\left(\mathbb{R}_{\tau} ; W^{1, q}(K)\right)\right),
$$

for all compact sets $K \subset \mathbb{R}^{n}$ and where $\mathcal{A} \equiv \mathcal{A}(t, \tau, \mathbf{x})$ is $\theta$-periodic in $\tau$.

We have

THEOREM 1.5 Under the assumptions above, if moreover the sequence $\left(u^{\varepsilon}\right)$ of solutions of (1.24) satisfies

$$
\left\|u^{\varepsilon}\right\|_{L^{\infty}\left(0, T ; L^{p}(\mathcal{O})\right)} \leq C,
$$


for some $p>1$ such that $\frac{1}{p}+\frac{1}{q^{\prime}}<1$, where $\frac{1}{q^{\prime}}=\operatorname{Max}\left\{\frac{1}{q}-\frac{1}{n}, 0\right\}$; then, extracting a subsequence,

$$
u^{\varepsilon} \text { 2-scale converges to a profile } U \in L^{\infty}\left(0, T ; L_{\theta}^{\infty}\left(\mathbb{R}_{\tau} ; L^{p}(\mathcal{O})\right)\right) \text {. }
$$

Moreover, we have

$$
U(t, \tau, \mathbf{x})=U_{0}\left(e^{-\tau M}(\mathbf{x}-\bar{N})+\bar{N}\right),
$$

where $\bar{N}$ is such that $-M \bar{N}=N$ and where $U_{0} \equiv U_{0}(t, \mathbf{y})$ is solution of

$$
\begin{aligned}
& \frac{\partial U_{0}}{\partial t}+\int_{0}^{\theta} e^{-\sigma M} \mathcal{A}\left(t, \sigma, e^{\sigma M}(\mathbf{y}-\bar{N})+\bar{N}\right) d \sigma \cdot \nabla_{y} U_{0}=0, \\
& U_{0 \mid t=0}=\frac{1}{\theta} u_{0},
\end{aligned}
$$

The proof of this Theorem consists in finding the constraint equation imposed on $U$ by the $\frac{1}{\varepsilon} L$ operator. This yields (1.28). Then we remove the essential oscillation of $u^{\varepsilon}$ by defining $w^{\varepsilon}(t, \mathbf{y})^{\varepsilon}=$ $\left.u^{\varepsilon}\left(t, e^{\frac{t}{\varepsilon} M}(\mathbf{y}-\bar{N})+\bar{N}\right)\right)$. Using the equation $w^{\varepsilon}$ satisfies, denoting $\left(W_{0}^{1, r}(K)\right)^{*}$ the dual of $W_{0}^{1, r}(K)$, we prove that $\frac{\partial w^{\varepsilon}}{\partial t}$ is bounded in $L^{\infty}\left(0, T ;\left(W_{0}^{1, r}(K)\right)^{*}\right)$, for some $r>1\left(\frac{1}{r^{*}}=\frac{1}{p}+\frac{1}{q}-\frac{1}{n}, \frac{1}{r}+\frac{1}{r^{*}}=1\right)$, which, applying the Aubin-Lions Lemma gives that $w^{\varepsilon} \rightarrow \theta U_{0}$ strongly in $L^{\infty}\left(0, T ;\left(W_{0}^{1, q}(K)\right)^{*}\right)$, for any compact set $K \subset \mathbb{R}^{n}$. This fact, coupled with (1.25), enables to pass to the limit in the equation satisfied by $w^{\varepsilon}$ and find (1.29).

Theorem 1.4 is a direct application of Theorem 1.5 once the wanted regularity of $\mathbf{E}^{\varepsilon}$ is proved. This is done with the help of classical kinetic energy estimates and regularization property of the Laplace operator.

The paper is organized as follow. In section 2 we present the scaling leading to the "Finite Larmor Radius Approximation". We show how to obtain equation (1.1) and system (1.17). The next section is devoted to the deduction of the asymptotic behavior of the linear Vlasov equation. Finally, in section 4 we prove Theorems 1.5 and 1.4 concerning the non-linear case.

Acknowledgments: We would like to thank Pierre Bertrand for the very interesting discutions we had with him and which helped us understand the "Finite Larmor Radius Approximation". We also thank Pierre-Arnaud Raviart for his pertinent advises about this work.

\section{Scaling: The Finite Larmor Radius regime}

We present here the scaling leading to equation (1.1) and system (1.17). We exhibit the important parameters playing a role when charged particles are submitted to a strong magnetic field. For this 
purpose we consider the following Vlasov-Poisson system

$$
\begin{aligned}
& \frac{\partial f}{\partial t}+\mathbf{v} \cdot \nabla_{x} f+\frac{q}{m}(\mathbf{E}(t, \mathbf{x})+\mathbf{v} \times \mathbf{B}(t, \mathbf{x})) \cdot \nabla_{v} f=0 \\
& f_{\mid t=0}=f_{0}, \\
& \mathbf{E}=-\nabla \phi, \quad-\Delta \phi=\frac{q}{\varepsilon_{0}} \rho, \\
& \rho(t, \mathbf{x})=\int_{\mathbb{R}_{v}^{3}} f(t, \mathbf{x}, \mathbf{v}) d \mathbf{v},
\end{aligned}
$$

before any scaling, which can be considered as a natural model to describe the behavior of charged particles under the action of an external magnetic field $\mathbf{B}(t, \mathbf{x})$.

We define some characteristic scales: $\bar{t}$ stands for a characteristic time, $\overline{L_{\|}}$for a characteristic length in the direction of the magnetic field, $\overline{L_{\perp}}$ for a characteristic length in the direction orthogonal to the magnetic field, $\bar{v}$ for a characteristic velocity. Denoting, for any vector $\mathbf{x}, \mathbf{x}_{\|}$and $\mathbf{x}_{\perp}$ its components parallel and perpendicular to the magnetic field, we now define new variables $t^{\prime} \mathbf{x}^{\prime}$ and $\mathbf{v}^{\prime}$, by $t=\bar{t} t^{\prime}, \mathbf{x}_{\|}=\overline{L_{\|}} \mathbf{x}_{\|}^{\prime}, \mathbf{x}_{\perp}=\overline{L_{\perp}} \mathbf{x}_{\perp}^{\prime}$, and $\mathbf{v}=\bar{v} \mathbf{v}^{\prime}$, making the characteristic scales the unities. In the same way, we define the scaling factors for the fields: $\bar{E}$ for the electric field and $\bar{B}$ for the magnetic field and the new fields $\mathcal{E}$ and $\mathcal{B}$ are given by: $\bar{E} \mathcal{E}\left(t^{\prime}, \mathbf{x}^{\prime}\right)=\mathbf{E}\left(\bar{t} t^{\prime}, \overline{L_{\|}} \mathbf{x}_{\|}^{\prime}, \overline{L_{\perp}} \mathbf{x}_{\perp}^{\prime}\right)$ and $\bar{B} \mathcal{B}\left(t^{\prime}, \mathbf{x}^{\prime}\right)=\mathbf{B}\left(\bar{t} t^{\prime}, \overline{L_{\|}} \mathbf{x}_{\|}^{\prime}, \overline{L_{\perp}} \mathbf{x}_{\perp}^{\prime}\right)$. Lastly, defining a scaling factor $\bar{f}$ for the repartition function, noticing that $f$ is a repartition function on the phase-space it is natural to define the new repartition function by

$$
\bar{f} f^{\prime}\left(t^{\prime}, \mathbf{x}^{\prime}, \mathbf{v}^{\prime}\right)=\overline{L_{\|}}{\overline{L_{\perp}}}^{2} \bar{v}^{3} f\left(\bar{t} t^{\prime}, \overline{L_{\|}} \mathbf{x}_{\|}^{\prime}, \overline{L_{\perp}} \mathbf{x}_{\perp}^{\prime}, \bar{v} \mathbf{v}^{\prime}\right) .
$$

With those new variables and fields we deduce the scaling equations.

\subsection{Scaling of the Vlasov equation}

Let us begin with the Vlasov equation, we obtain that $f^{\prime}$ is solution of

$$
\frac{\partial f^{\prime}}{\partial t^{\prime}}+\frac{\bar{v} \bar{t}}{\overline{L_{\|}}} \mathbf{v}_{\|}^{\prime} \cdot \nabla_{x^{\prime}} f^{\prime}+\frac{\bar{v} \bar{t}}{\overline{L_{\perp}}} \mathbf{v}_{\perp}^{\prime} \cdot \nabla_{x^{\prime}} f^{\prime}+\left(\frac{q \bar{E} \bar{t}}{m \bar{v}} \mathcal{E}\left(t^{\prime}, \mathbf{x}^{\prime}\right)+\frac{q \bar{B} \bar{t}}{m} \mathbf{v}^{\prime} \times \mathcal{B}\left(t^{\prime}, \mathbf{x}^{\prime}\right)\right) \cdot \nabla_{v^{\prime}} f^{\prime}=0
$$

Now, we introduce the characteristic cyclotron frequency: $\bar{\omega}_{c}=\frac{q \bar{B}}{m}$ and the characteristic Larmor radius: $\bar{a}_{L}=\frac{\bar{v}}{\bar{\omega}_{c}}$. Using those physical quantities, (2.3) becomes

$$
\frac{\partial f^{\prime}}{\partial t^{\prime}}+\bar{t} \bar{\omega}_{c} \frac{\bar{a}_{L}}{\bar{L}_{\|}} \mathbf{v}_{\|}^{\prime} \cdot \nabla_{x^{\prime}} f^{\prime}+\bar{t}_{\bar{\omega}_{c}} \frac{\bar{a}_{L}}{\bar{L}_{\perp}} \mathbf{v}_{\perp}^{\prime} \cdot \nabla_{x^{\prime}} f^{\prime}+\left(\bar{t} \bar{\omega}_{c} \frac{\bar{E}}{\bar{v} \bar{B}} \mathcal{E}\left(t^{\prime}, \mathbf{x}^{\prime}\right)+\bar{t} \bar{\omega}_{c} \mathbf{v}^{\prime} \times \mathcal{B}\left(t^{\prime}, \mathbf{x}^{\prime}\right)\right) \cdot \nabla_{v^{\prime}} f^{\prime}=0
$$

Assuming the magnetic field is strong consists essentially in setting

$$
\bar{t} \overline{\omega_{c}}=\frac{1}{\varepsilon} \text { and } \frac{\bar{E}}{\bar{v} \bar{B}}=\varepsilon
$$

for a small parameter $\varepsilon$, and, the "Finite Larmor Radius" regime consists in choosing

$$
\frac{\bar{a}_{L}}{\overline{L_{\|}}}=\varepsilon \text { and } \frac{\bar{a}_{L}}{\overline{L_{\perp}}}=1
$$


Hence the rescaled Vlasov equation writes

$$
\frac{\partial f^{\prime}}{\partial t^{\prime}}+\mathbf{v}_{\|}^{\prime} \cdot \nabla_{x^{\prime}} f^{\prime}+\frac{1}{\varepsilon} \mathbf{v}_{\perp}^{\prime} \cdot \nabla_{x^{\prime}} f^{\prime}+\left(\mathcal{E}\left(t^{\prime}, \mathbf{x}^{\prime}\right)+\frac{1}{\varepsilon} \mathbf{v} \times \mathcal{B}\left(t^{\prime}, \mathbf{x}^{\prime}\right)\right) \cdot \nabla_{v^{\prime}} f^{\prime}=0 .
$$

Concerning the initial data, under the scaling (2.2), (2.1.b) directly gives

$$
f_{\mid t^{\prime}=0}^{\prime}=\frac{\overline{L_{\|}}{\overline{L_{\perp}}}^{2} \bar{v}^{3}}{\bar{f}} f_{0}\left(\overline{L_{\|}} \mathbf{x}_{\|}^{\prime}, \overline{L_{\perp}} \mathbf{x}_{\perp}^{\prime}, \bar{v} \mathbf{v}^{\prime}\right)
$$

Hence, if we assume that the scales of variations of the initial data $f_{0}$ (before scaling) is of the same order than the characteristic lengths used, and that $\bar{f}={\overline{L_{\|}}}_{L_{\perp}}^{2} \bar{v}^{3}$, it is natural to consider equation (1.1) as a relevant model to understand local behavior of charged particles under the action of a strong external constant magnetic field.

This is the reason why we study (1.1) in section 3 .

\subsection{Scaling of the Poisson equation}

We now turn to the Poisson equation (2.1.c),(2.1.d). For this purpose, we define the new electric potential by

$$
\bar{E} \overline{L_{\|}} \phi^{\prime}\left(t^{\prime}, \mathbf{x}^{\prime}\right)=\phi\left(\bar{t} t^{\prime}, \overline{L_{\|}} \mathbf{x}_{\|}^{\prime}, \overline{L_{\perp}} \mathbf{x}_{\perp}^{\prime}\right),
$$

and the new particle density by

$$
\rho^{\prime}\left(t^{\prime}, \mathbf{x}^{\prime}\right)=\int f^{\prime}\left(t^{\prime}, \mathbf{x}^{\prime}, \mathbf{v}^{\prime}\right) d \mathbf{v}^{\prime}
$$

Direct computations give:

$$
\begin{gathered}
\rho^{\prime}\left(t^{\prime}, \mathbf{x}^{\prime}\right)=\frac{\overline{L_{\|}}{\overline{L_{\perp}}}^{2}}{\bar{f}} \rho\left(\bar{t} t^{\prime}, \overline{L_{\|}} \mathbf{x}_{\perp}^{\prime}, \overline{L_{\perp}} \mathbf{x}_{\|}^{\prime}\right), \\
\nabla \phi\left(\bar{t} t^{\prime}, \overline{L_{\|}} \mathbf{x}_{\perp}^{\prime}, \overline{L_{\perp}} \mathbf{x}_{\|}^{\prime}\right)=\bar{E}\left(\begin{array}{c}
\nabla_{\mathbf{x}_{\|\|}^{\prime}} \phi^{\prime}\left(t^{\prime}, \mathbf{x}^{\prime}\right) \\
\frac{\overline{L_{\|}}}{\overline{L_{\perp}}} \nabla_{\mathbf{x}_{\perp}^{\prime}} \phi^{\prime}\left(t^{\prime}, \mathbf{x}^{\prime}\right)
\end{array}\right),
\end{gathered}
$$

and

$$
\Delta \phi\left(\bar{t} t^{\prime}, \overline{L_{\|}} \mathbf{x}_{\|}^{\prime}, \overline{L_{\perp}} \mathbf{x}_{\perp}^{\prime}\right)=\frac{\bar{E}}{\overline{L_{\|}}}\left(\Delta_{\mathbf{x}_{\|}^{\prime}} \phi^{\prime}\left(t^{\prime}, \mathbf{x}^{\prime}\right)+\frac{{\overline{L_{\|}}}^{2}}{{\overline{L_{\perp}}}^{2}} \Delta_{\mathbf{x}_{\perp}^{\prime}} \phi^{\prime}\left(t^{\prime}, \mathbf{x}^{\prime}\right)\right) .
$$

Hence the Poisson equation $-\Delta \phi=\frac{q}{\varepsilon_{0}} \rho$ becomes

$$
-\left(\Delta_{\mathbf{x}_{\|}^{\prime}} \phi^{\prime}+\frac{{\overline{L_{\|}}}^{2}}{{\overline{L_{\perp}}}^{2}} \Delta_{\mathbf{x}_{\perp}^{\prime}} \phi^{\prime}\right)=\frac{q}{\varepsilon_{0}} \frac{\bar{f}}{\bar{E}{\overline{L_{\perp}}}^{2}} \rho^{\prime}
$$

and the definition of the electric field $\mathbf{E}=-\nabla \phi$ yields

$$
\mathcal{E}=-\left(\begin{array}{c}
\nabla_{\mathbf{x}_{\|}^{\prime}} \phi^{\prime} \\
\overline{\bar{L}_{\|}} \\
\overline{L_{\perp}} \nabla_{\mathbf{x}_{\perp}^{\prime}} \phi^{\prime}
\end{array}\right) \text {. }
$$

$\mathrm{RR} \mathrm{n}^{\circ} 3847$ 
Setting now the same ratio as in (2.5) and (2.6) and considering that the scales of variations of the initial data is of the same order than the characteristic lengths, the rescaled Vlasov-Poisson system writes

$$
\begin{aligned}
& \frac{\partial f^{\prime}}{\partial t^{\prime}}+\mathbf{v}_{\|}^{\prime} \cdot \nabla_{x^{\prime}} f^{\prime}+\frac{1}{\varepsilon} \mathbf{v}_{\perp}^{\prime} \cdot \nabla_{x^{\prime}} f^{\prime}+\left(\mathcal{E}\left(t^{\prime}, \mathbf{x}^{\prime}\right)+\frac{1}{\varepsilon} \mathbf{v} \times \mathcal{B}\left(t^{\prime}, \mathbf{x}^{\prime}\right)\right) \cdot \nabla_{v^{\prime}} f^{\prime}=0, \\
& f_{\mid t=0}=f_{0}^{\prime}, \\
& \mathcal{E}=-\left(\begin{array}{c}
\nabla_{\mathbf{x}_{\|}^{\prime}} \phi^{\prime} \\
\frac{1}{\varepsilon} \nabla_{\mathbf{x}_{\perp}^{\prime}} \phi^{\prime}
\end{array}\right),-\left(\Delta_{\mathbf{x}_{\|}^{\prime}} \phi^{\prime}+\frac{1}{\varepsilon^{2}} \Delta_{\mathbf{x}_{\perp}^{\prime}} \phi^{\prime}\right)=\gamma \rho^{\prime}, \\
& \rho^{\prime}\left(t^{\prime}, \mathbf{x}^{\prime}\right)=\int_{\mathbb{R}_{v}^{3}} f^{\prime}\left(t^{\prime}, \mathbf{x}^{\prime}, \mathbf{v}^{\prime}\right) d \mathbf{v}^{\prime},
\end{aligned}
$$

with $\gamma=\frac{q}{\varepsilon_{0}} \frac{\bar{f}}{E L_{\perp}}$.

For the study we lead in section 4 we consider the previous system with $\gamma=\frac{1}{\varepsilon}$ and with $\mathcal{B}=\mathcal{M}=$ $\mathbf{e}_{1}$. We moreover assume that none of the fields depend on the component parallel to the magnetic fields $\mathbf{x}_{\|}$and $\mathbf{v}_{\|}$. In this case the Poisson equation from which we remove the $\mathbf{x}_{\|}$-dependency

$$
\mathcal{E}=-\left(\begin{array}{c}
0 \\
\frac{1}{\varepsilon} \nabla_{\mathbf{x}_{\perp}^{\prime}} \phi^{\prime}
\end{array}\right),-\frac{1}{\varepsilon^{2}} \Delta_{\mathbf{x}_{\perp}^{\prime}} \phi^{\prime}=\frac{1}{\varepsilon} \rho^{\prime}
$$

is equivalent to, removing the magnetic field direction,

$$
\mathcal{E}=-\nabla \phi^{*},-\Delta \phi^{*}=\rho^{*},
$$

where $\phi^{*}$ is nothing but $\frac{1}{\varepsilon} \phi^{\prime}$ and with

$$
\rho^{*}=\int_{\mathbb{R}_{v}^{2}} f^{\prime} d \mathbf{v},
$$

explaining the interest of studying system (1.17).

\section{Homogenization of the Vlasov equation}

In this section, we provide the homogenization of the Vlasov equation (1.1) and prove Theorem 1.1. Since the contexts of equation (1.1) and the equation studied in E. Frénod \& E. Sonnendrücker [9] are similar we develop a generic framework and apply it to prove Theorem 1.1. We then show that this generic framework applies also to prove Theorems 1.1 and 3.2 of E. Frénod \& E. Sonnendrücker [9].

\subsection{Generic framework - Proof of Theorem 1.3}

The framework inside which the problem we want to homogenize enters is the following conservation law singularly linearly perturbed

$$
\begin{aligned}
& \frac{\partial u^{\varepsilon}}{\partial t}+\mathbf{A} \cdot \nabla_{x} u^{\varepsilon}+\frac{1}{\varepsilon} \mathbf{L} \cdot \nabla_{x} u^{\varepsilon}=0 \\
& u_{\mid t=0}^{\varepsilon}=u_{0}
\end{aligned}
$$


where $u^{\varepsilon} \equiv u^{\varepsilon}(t, \mathbf{x}), t \in[0, T)$ for some $T<\infty$ and $\mathbf{x} \in \mathbb{R}^{n}=\mathcal{O}$. We denote $\mathcal{Q}=[0, T) \times \mathcal{O}$, and we assume $\mathbf{A} \equiv \mathbf{A}(t, \mathbf{x}) \in L^{\infty}\left(0, T ; L_{l o c}^{2}(\mathcal{O})\right)$, with $\nabla_{x} \cdot \mathbf{A}=0$ and $\mathbf{L} \equiv M \mathbf{x}+N$ where $M$ is a real $n \times n$ matrix with constant entries satisfying $\operatorname{tr} M=0$ and where $N \in \operatorname{Im} M$. We moreover assume that $e^{\tau M}$ is $\theta$-periodic for a given $\theta \in \mathbb{R}$.

The proof of Theorem 1.3, characterizing the limit of equation (3.1), is led in three steps. First, we look for the constraint imposed by the operator $\left(\frac{1}{\varepsilon} \mathbf{L} \cdot \nabla_{x}\right)$ on the profile $U, 2$-scale limit of $\left(u^{\varepsilon}\right)$. Studying the characteristics associated with this constraint, we obtain the form (1.14) it gives to $U$. In the second step, using test functions satisfying the constraint in the weak formulation of (3.1), we get the equation satisfied by $U_{0}$. In view of formula (1.8) linking the the 2 -scale limit to the weak-* limit, in the last step, we integrate the equation satisfied by $U_{0}$ to deduce (1.16).

Under the assumption (1.11), we may apply the result of N'Guetseng [17] and Allaire [2] (see Theorem 1.2). Then, for any period $\tilde{\theta}$ there exists a $\tilde{\theta}$-periodic profile $U_{\tilde{\theta}}(t, \tau, \mathbf{x}) \in L^{\infty}\left(0, T ; L_{\tilde{\theta}}^{\infty}\left(\mathbb{R}_{\tau} ; L^{2}(\mathcal{O})\right)\right)$ such that, for any regular function $\psi_{\tilde{\theta}}(t, \tau, \mathbf{x})$ compactly supported in $(t, \mathbf{x})$ and $\tilde{\theta}$-periodic in $\tau$, we have

$$
\int_{\mathcal{Q}} u^{\varepsilon}(t, \mathbf{x}) \psi_{\tilde{\theta}}\left(t, \frac{t}{\varepsilon}, \mathbf{x}\right) d t d \mathbf{x} \rightarrow \int_{\mathcal{Q}} \int_{0}^{\tilde{\theta}} U(t, \tau, \mathbf{x}) \psi_{\tilde{\theta}}(t, \tau, \mathbf{x}) d \tau d t d \mathbf{x} .
$$

Now, we write a weak formulation of (3.1) with oscillating test functions $\left(\psi_{\tilde{\theta}}\right)^{\varepsilon}=\psi_{\tilde{\theta}}\left(t, \frac{t}{\varepsilon}, \mathbf{x}\right)$, with $\psi_{\tilde{\theta}}(t, \tau, \mathbf{x})$ previously defined. Since $\nabla_{x} \cdot \mathbf{A}=\nabla_{x} \cdot \mathbf{L}=0$, it writes

$$
\int_{\mathcal{Q}} u^{\varepsilon}\left(\left(\frac{\partial \psi_{\tilde{\theta}}}{\partial t}\right)^{\varepsilon}+\frac{1}{\varepsilon}\left(\frac{\partial \psi_{\tilde{\theta}}}{\partial \tau}\right)^{\varepsilon}+\mathbf{A} \cdot\left(\nabla_{x} \psi_{\tilde{\theta}}\right)^{\varepsilon}+\frac{1}{\varepsilon} \mathbf{L} \cdot\left(\nabla_{x} \psi_{\tilde{\theta}}\right)^{\varepsilon}\right) d t d \mathbf{x}=-\int_{\mathcal{O}} u_{0} \psi_{\tilde{\theta}}(0,0, \mathbf{x}) d \mathbf{x} .
$$

Multiplying (3.3) by $\varepsilon$ and passing to the limit gives the following constraint equation for the $\tilde{\theta}$ periodic profile $U_{\tilde{\theta}}$ :

$$
\frac{\partial U_{\tilde{\theta}}}{\partial \tau}+\mathbf{L} \cdot \nabla_{x} U_{\tilde{\theta}}=0 \text { in } \mathcal{D}^{\prime}\left(\mathbb{R}_{\tau} \times \mathcal{O}\right)
$$

This equation says that $U_{\tilde{\theta}}$ is constant along the characteristics of the following dynamical system

$$
\frac{d \mathbf{X}}{d \tau}=\mathbf{L}(\mathbf{X}(\tau))=M \mathbf{X}(\tau)+N .
$$

Using the assumptions made on $\mathbf{L}$, writing $\mathbf{X}(\tau ; \mathbf{x}, s)$ for the solution of (3.5) satisfying $\mathbf{X}(s ; \mathbf{x}, s)=\mathbf{x}$, we obtain

$$
\mathbf{X}(\tau ; \mathbf{x}, s)=e^{(\tau-s) M}(\mathbf{x}-\bar{N})+\bar{N} .
$$

Hence from equation (3.4), we deduce, on the one hand, that for any $\tilde{\theta}$ the $\tilde{\theta}$-periodic profile writes

$$
U_{\tilde{\theta}}(t, \tau, \mathbf{x})=U_{0}\left(t, e^{-\tau M}(\mathbf{x}-\bar{N})+\bar{N}\right),
$$

for a function $U_{0} \equiv U_{0}(t, \mathbf{y}) \in L^{\infty}\left(0, T ; L^{2}\left(\mathcal{O}^{\prime}\right)\right)$. On the other hand, we take the $\theta$-periodicity of $e^{-\tau M}$ under consideration. In view of (3.7), we deduce that if $\tilde{\theta}$ and $\theta$ are incommensurable $U_{\tilde{\theta}}$ cannot depend on $\tau$, and then contains no information concerning the oscillations of $\left(u^{\varepsilon}\right)$. Yet, if $\tilde{\theta}$ equals (or is multiple of) $\theta$ the profile $U_{\tilde{\theta}}$ naturally satisfies its $\tilde{\theta}$-periodicity condition once (3.7) is satisfied.

$\mathrm{RR} \mathrm{n}^{\circ} 3847$ 
Hence, among every possible periodic profile, we are incited to work now with the $\theta$-periodic one

$$
U:=U_{\theta},
$$

which writes

$$
U(t, \tau, \mathbf{x})=U_{0}\left(t, e^{-\tau M}(\mathbf{x}-\bar{N})+\bar{N}\right),
$$

for $U_{0} \equiv U_{0}(t, \mathbf{y}) \in L^{\infty}\left(0, T ; L^{2}\left(\mathcal{O}^{\prime}\right)\right)$, which is the equality (1.14) of Theorem 1.3.

Now, we seek the equation $U_{0}$ satisfies. For this purpose, we build oscillating test functions satisfying the constraint and use them in the weak formulation (3.3).

For any $\varphi(t, \mathbf{y})$, regular and compactly supported, we define $\psi(t, \tau, \mathbf{x})=\varphi\left(t, e^{-\tau M}(\mathbf{x}-\bar{N})+\bar{N}\right)$ and we inject in (3.3) test function $(\psi)^{\varepsilon}=\psi\left(t, \frac{t}{\varepsilon}, \mathbf{x}\right)$. Acting in such a way, the terms containing the constraint vanishes. We have then

$$
\int_{\mathcal{Q}} u^{\varepsilon}\left(\left(\frac{\partial \psi}{\partial t}\right)^{\varepsilon}+\mathbf{A} \cdot\left(\nabla_{x} \psi\right)^{\varepsilon}\right) d t d \mathbf{x}=-\int_{\mathcal{O}} u_{0} \psi(0,0, \mathbf{x}) d \mathbf{x}
$$

which passing to the limit yields

$$
\int_{\mathcal{Q}} \int_{0}^{\theta} U\left(\frac{\partial \psi}{\partial t}+\mathbf{A} \cdot \nabla \psi\right) d t d \mathbf{x} d \tau=-\int_{\mathcal{O}} u_{0} \psi(0,0, \mathbf{x}) d \mathbf{x}
$$

In (3.11) we use the expression of $U$ in terms of $U_{0}$, the expression of $\psi$ in term of $\varphi$, without forgetting

$$
\nabla_{x} \psi(t, \tau, \mathbf{x})=\left(e^{-\tau M}\right)^{T} \nabla_{y} \varphi\left(t, e^{-\tau M}(\mathbf{x}-\bar{N})+\bar{N}\right),
$$

denoting $\left(e^{-\tau M}\right)^{T}$ the transpose of $e^{-\tau M}$; and we make the change of variable $\mathbf{x} \mapsto \mathbf{y}=e^{-\tau M}(\mathbf{x}-$ $\bar{N})+\bar{N}$. This gives

$$
\int_{\mathcal{Q}^{\prime}} \int_{0}^{\theta} U_{0}\left(\frac{\partial \varphi}{\partial t}+e^{-\tau M} \mathbf{A}\left(t, e^{\tau M}(\mathbf{y}-\bar{N})+\bar{N}\right) \cdot \nabla_{y} \varphi\right) d t d \mathbf{y} d \tau=-\int_{\mathcal{O}} u_{0} \varphi(0, \mathbf{y}) d \mathbf{y} .
$$

An easy computation coupled with the fact that $\nabla_{x} \cdot \mathbf{A}=0$ gives

$$
\nabla_{y} \cdot\left(e^{-\sigma M} \mathbf{A}\left(t, e^{\sigma M}(\mathbf{y}-\bar{N})+\bar{N}\right)\right)=\left(\nabla_{x} \cdot \mathbf{A}\right)\left(t, e^{\sigma M}(\mathbf{y}-\bar{N})+\bar{N}\right)=0 .
$$

Hence, knowing that neither $U_{0}$ nor $\varphi$ depend on $\tau$, we deduce that (3.13) is the weak formulation of

$$
\begin{aligned}
& \frac{\partial U_{0}}{\partial t}+\frac{1}{\theta} \int_{0}^{\theta} e^{-\sigma M} \mathbf{A}\left(t, e^{\sigma M}(\mathbf{y}-\bar{N})+\bar{N}\right) d \sigma \cdot \nabla_{y} U_{0}=0, \\
& U_{0 \mid t=0}=\frac{1}{\theta} u_{0},
\end{aligned}
$$

proving the second part of Theorem 1.3.

In order to get equation (1.16) we use the fact

$$
u(t, \mathbf{x})=\int_{0}^{\theta} U(t, \tau, \mathbf{x}) d \tau=\int_{0}^{\theta} U_{0}\left(t, e^{-\tau M}(\mathbf{x}-\bar{N})+\bar{N}\right) d \tau .
$$


Replacing in (3.15) y by $e^{-\tau M}(\mathbf{x}-\bar{N})+\bar{N}$ and integrating in $\tau$ we get

$$
\begin{aligned}
& \frac{\partial}{\partial t}\left(\int_{0}^{\theta} U_{0}\left(t, e^{-\tau M}(\mathbf{x}-\bar{N})+\bar{N}\right) d \tau\right)+ \\
& \quad \frac{1}{\theta} \int_{0}^{\theta} \int_{0}^{\theta} e^{-\sigma M} \mathbf{A}\left(t, e^{(\sigma-\tau) M}(\mathbf{x}-\bar{N})+\bar{N}\right) d \sigma \cdot \nabla_{y} U_{0}\left(t, e^{-\tau M}(\mathbf{x}-\bar{N})+\bar{N}\right) d \tau=0, \\
& \left.\int_{0}^{\theta} U_{0}\left(t, e^{-\tau M}(\mathbf{x}-\bar{N})+\bar{N}\right)\right)_{\mid t=0} d \tau=\frac{1}{\theta} \int_{0}^{\theta} u_{0}\left(e^{-\tau M}(\mathbf{x}-\bar{N})+\bar{N}\right) d \tau .
\end{aligned}
$$

An easy computation yields

$$
\nabla_{x}\left(U_{0}\left(t, e^{-\tau M}(\mathbf{x}-\bar{N})+\bar{N}\right)\right)=\left(e^{-\tau M}\right)^{T}\left(\nabla_{y} U_{0}\right)\left(t, e^{-\tau M}(\mathbf{x}-\bar{N})+\bar{N}\right),
$$

and then replacing in the second term of (3.17.a) gives

$$
\begin{aligned}
\int_{0}^{\theta} & \int_{0}^{\theta} e^{-\sigma M} \mathbf{A}\left(t, e^{(\sigma-\tau) M}(\mathbf{x}-\bar{N})+\bar{N}\right) d \sigma \cdot\left(e^{\tau M}\right)^{T} \nabla_{x}\left(U_{0}\left(t, e^{-\tau M}(\mathbf{x}-\bar{N})+\bar{N}\right) d \tau\right. \\
& =\int_{0}^{\theta} \int_{0}^{\theta} e^{(\tau-\sigma) M} \mathbf{A}\left(t, e^{(\sigma-\tau) M}(\mathbf{x}-\bar{N})+\bar{N}\right) d \sigma \cdot \nabla_{x}\left(U_{0}\left(t, e^{-\tau M}(\mathbf{x}-\bar{N})+\bar{N}\right)\right) d \tau .
\end{aligned}
$$

Yet, by the periodicity of $\tau \mapsto e^{\tau M}$ we deduce that

$$
\int_{0}^{\theta} e^{(\tau-\sigma) M} \mathbf{A}\left(t, e^{(\sigma-\tau) M}(\mathbf{x}-\bar{N})+\bar{N}\right) d \sigma=\int_{0}^{\theta} e^{-\sigma M} \mathbf{A}\left(t, e^{\sigma M}(\mathbf{x}-\bar{N})+\bar{N}\right) d \sigma,
$$

does not depend on $\tau$.

We may finally conclude that (3.17) reads

$$
\begin{aligned}
& \frac{\partial u}{\partial t}+\frac{1}{\theta} \int_{0}^{\theta} e^{-\sigma M} \mathbf{A}\left(t, e^{\sigma M}(\mathbf{x}-\bar{N})+\bar{N}\right) d \sigma \cdot \nabla u=0, \\
& u_{\mid t=0}(\mathbf{x})=\frac{1}{\theta} \int_{0}^{\theta} u_{0}\left(e^{-\sigma M}(\mathbf{x}-\bar{N})+\bar{N}\right) d \sigma .
\end{aligned}
$$

achieving the proof of Theorem 1.3.

\subsection{Application to the Vlasov equation - Proof of Theorem 1.1}

Using assumption (1.2) made on $f_{0}$ and the following property of $f^{\varepsilon}$ solution of (1.1):

$$
\frac{d}{d t}\left\|f^{\varepsilon}(t, \cdot, \cdot)\right\|_{L^{2}(\mathcal{O})}=0
$$

obtained by a direct integration in $\mathbf{x}$ and $\mathbf{v}$ of (1.1.a), after multiplication by $f^{\varepsilon}$, we deduce

$$
\left\|f^{\varepsilon}\right\|_{L^{\infty}\left(0, T ; L^{2}(\mathcal{O})\right)} \leq C
$$

for some constants $C$.

Hence, the Vlasov equation (1.1) enters the generic framework previously built with

$$
\mathbf{A}(t, \mathbf{x}, \mathbf{v})=\left(\begin{array}{c}
\mathbf{v}_{\|} \\
\mathbf{E}(t, \mathbf{x})
\end{array}\right)\left(\in \mathbb{R}^{6}\right) \text { and } \mathbf{L}(t, \mathbf{x}, \mathbf{v})=\left(\begin{array}{c}
\mathbf{v}_{\perp} \\
\mathbf{v} \times \mathcal{M}
\end{array}\right)\left(\in \mathbb{R}^{6}\right) .
$$

$\mathrm{RR} \mathrm{n}^{\circ} 3847$ 
In this case $e^{\tau M}$ becomes

$$
e^{\tau M}=\left(\begin{array}{ll}
I & \mathcal{R}(\tau) \\
0 & R(\tau)
\end{array}\right)
$$

with $\mathcal{R}(\tau)$ and $R(\tau)$ given by (1.6).

We can then deduce

$$
f^{\varepsilon} \text { 2-scale converges to } F \in L^{\infty}\left(0, T ; L_{2 \pi}^{\infty}\left(\mathbb{R}_{\tau} ; L^{2}(\mathcal{O})\right)\right),
$$

and applying Theorem 1.3, we can deduce that there exists a function $G \equiv G(t, \mathbf{y}, \mathbf{u}) \in L^{\infty}\left(0, T ; L^{2}\left(\mathcal{O}^{\prime}\right)\right)$ such that

$$
F(t, \tau, \mathbf{x}, \mathbf{v})=G(t, \mathbf{x}+\mathcal{R}(-\tau) \mathbf{v}, R(-\tau) \mathbf{v})
$$

where $G$ is the unique solution of

$$
\begin{aligned}
& \frac{\partial G}{\partial t}+\mathbf{u}_{\|} \cdot \nabla_{y} G+\frac{1}{2 \pi}\left(\int_{0}^{2 \pi} \mathcal{R}(-\tau) \mathbf{E}(t, \mathbf{y}+\mathcal{R}(\tau) \mathbf{u}) d \tau\right) \cdot \nabla_{y} G \\
& +\frac{1}{2 \pi}\left(\int_{0}^{2 \pi} R(-\tau) \mathbf{E}(t, \mathbf{y}+\mathcal{R}(\tau) \mathbf{u}) d \tau\right) \cdot \nabla_{v} G=0, \\
& G_{\mid t=0}=\frac{1}{2 \pi} f_{0} .
\end{aligned}
$$

Always applying Theorem 1.3, we also deduce that the weak-* limit $f$ of $\left(f^{\varepsilon}\right)$ is the unique solution of (1.5).

The fact that the whole sequence $\left(f^{\varepsilon}\right) 2$-scale converges to $F$ and weak-* converges to $f$ is a direct consequence of the uniqueness of the solution of (3.28) and (1.5). This ends the proof of Theorem 1.1.

\subsection{Link with physical models}

In order to compare the model we obtain with the "Finite Larmor Radius Approximation" used by physicists, we restrain to the plane orthogonal to the magnetic field. Denoting here $R(\tau)$ and $\mathcal{R}(\tau)$ there restrictions to this plan, we introduce the Larmor Radius variable: $\mathbf{r}=\mathbf{v}^{\perp}$ and the guiding center variable: $\mathbf{x}_{C}=\mathbf{x}-\mathbf{r}$ where for any vector $\mathbf{v}=\left(v_{2}, v_{3}\right), \mathbf{v}^{\perp}$ stand for $\mathbf{v}^{\perp}=\left(-v_{3}, v_{2}\right)$. In this new variables, equation (3.28) reads:

$$
\frac{\partial f}{\partial t}-\frac{1}{2 \pi}\left(\int_{0}^{2 \pi} \mathbf{E}^{\perp}\left(t, \mathbf{x}_{C}+R(\tau) \mathbf{r}\right) d \tau\right) \cdot \nabla_{x_{C}} f+\frac{1}{2 \pi}\left(\int_{0}^{2 \pi} R(-\tau) \mathbf{E}^{\perp}\left(t, \mathbf{x}_{C}+R(\tau) \mathbf{r}\right) d \tau\right) \cdot \nabla_{r} f=0 .
$$

Assuming that the distribution function is a Maxwellian distribution, i.e. $f \equiv n\left(\mathbf{x}_{c}, t\right) e^{-\mathbf{r}^{2} /\left(2 \sigma^{2}\right)} /(2 \pi \sigma)$, we integrate (3.29) with respect to $\mathbf{r}$. This procedure cancels the third term. Indeed

$$
\int_{0}^{2 \pi} R(-\tau) \mathbf{E}^{\perp}\left(t, \mathbf{x}_{C}+R(\tau) \mathbf{r}\right) d \tau
$$

only depends on $|\mathbf{r}|$ and then the integrand is odd. Then we get

$$
\frac{\partial n}{\partial t}-\int_{\mathbb{R}_{r}^{2}} \frac{1}{2 \pi}\left(\int_{0}^{2 \pi} \mathbf{E}^{\perp}\left(t, \mathbf{x}_{C}+R(\tau) \mathbf{r}\right) d \tau\right) e^{-\mathbf{r}^{2} /\left(2 \sigma^{2}\right)} /(2 \pi \sigma) d \mathbf{r} \cdot \nabla_{x_{C}} n=0,
$$

which is the model introduced by Hansen et al. [14]. 


\subsection{About previous results}

Notice that Theorem 1.1 of E. Frénod \& E. Sonnendrücker [9], proving that the asymptotic behavior of

$$
\begin{aligned}
& \frac{\partial f^{\varepsilon}}{\partial t}+\mathbf{v} \cdot \nabla_{x} f^{\varepsilon}+\left(\mathbf{E}+\mathbf{v} \times\left(\mathbf{B}+\frac{\mathcal{M}}{\varepsilon}\right)\right) \cdot \nabla_{v} f^{\varepsilon}=0, \\
& f_{\mid t=0}^{\varepsilon}=f_{0},
\end{aligned}
$$

is given by

$$
\begin{aligned}
& \frac{\partial f}{\partial t}+\mathbf{v}_{\|} \cdot \nabla_{x} f+\left(\mathbf{E}_{\|}+\mathbf{v} \times \mathbf{B}_{\|}\right) \cdot \nabla_{v} f=0 \\
& f_{\mid t=0}=\frac{1}{2 \pi} \int_{0}^{2 \pi} f_{0}(\mathbf{x}, \mathbf{u}(\mathbf{v}, \tau)) d \tau
\end{aligned}
$$

is also a consequence of Theorem 1.3 by setting

$$
\mathbf{A}=\left(\begin{array}{c}
\mathbf{v} \\
\mathbf{E}+\mathbf{v} \times \mathbf{B}
\end{array}\right) \text { and } \mathbf{L}=\left(\begin{array}{c}
0 \\
\mathbf{v} \times \mathcal{M}
\end{array}\right)
$$

This is the same for Theorem 3.2 of [9] with

$$
\mathbf{A}=\left(\begin{array}{c}
\mathbf{v} \\
\mathbf{E}+\mathbf{v} \times \mathbf{B}
\end{array}\right) \text { and } \mathbf{L}=\left(\begin{array}{c}
0 \\
\mathcal{N}+\mathbf{v} \times \mathcal{M}
\end{array}\right)
$$

This Theorem says that the weak-* limit of the solution of

$$
\begin{aligned}
& \frac{\partial f^{\varepsilon}}{\partial t}+\mathbf{v} \cdot \nabla_{x} f^{\varepsilon}+\left(\left(\mathbf{E}+\frac{\mathcal{N}}{\varepsilon}\right)+\mathbf{v} \times\left(\mathbf{B}+\frac{\mathcal{M}}{\varepsilon}\right)\right) \cdot \nabla_{v} f^{\varepsilon}=0, \\
& f_{\mid t=0}^{\varepsilon}=f_{0},
\end{aligned}
$$

with $\mathcal{N}=\mathbf{e}_{2}$, satisfies

$$
\begin{aligned}
& \frac{\partial f}{\partial t}+\left(\begin{array}{c}
v_{1} \\
0 \\
-1
\end{array}\right) \cdot \nabla_{x} f+\left[\left(\begin{array}{c}
E_{1}-B_{2} \\
0 \\
0
\end{array}\right)+\left(\begin{array}{c}
v_{1} \\
v_{2} \\
v_{3}+1
\end{array}\right) \times\left(\begin{array}{c}
B_{1} \\
0 \\
0
\end{array}\right)\right] \cdot \nabla_{v} f=0 \\
& f_{\mid t=0}=\frac{1}{2 \pi} \int_{0}^{2 \pi} f_{0}(\mathbf{x}, \mathbf{u}(\mathbf{v}, \tau)) d \tau
\end{aligned}
$$

\section{2-scale limit of the 2D Vlasov-Poisson system}

The aim of this section is to characterize the equation satisfied by the 2-scale limit of the sequence $\left(f^{\varepsilon}, \mathbf{E}^{\varepsilon}\right)$ of the Vlasov-Poisson system (1.17) For this purpose, we generalize the generic framework to the case when the operator $\mathbf{A}^{\varepsilon}$ is oscillating. Then, we apply the results obtained in this new generic framework to prove Theorem 1.4.

$\mathrm{RR} \mathrm{n}^{\circ} 3847$ 


\subsection{Generalized generic framework - Proof of Theorem 1.5}

We consider here

$$
\begin{aligned}
& \frac{\partial u^{\varepsilon}}{\partial t}+\mathbf{A}^{\varepsilon} \cdot \nabla_{x} u^{\varepsilon}+\frac{1}{\varepsilon} \mathbf{L} \cdot \nabla_{x} u^{\varepsilon}=0, \\
& u_{t=0}^{\varepsilon}=u_{0} .
\end{aligned}
$$

where the notations are the same as for equation (1.10): $u^{\varepsilon} \equiv u^{\varepsilon}(t, \mathbf{x}), t \in[0, T), T<\infty ; \mathbf{x} \in \mathbb{R}^{n}=$ $\mathcal{O}, \mathcal{Q}=[0, T) \times \mathcal{O}$. We suppose, as previously, that $\mathbf{L} \equiv M \mathbf{x}+N$, where $M$ is a constant entry matrix satisfying $\operatorname{tr} M=0$ and $e^{\tau M}$ is $\theta$-periodic. The assumptions we make on $\mathbf{A}^{\varepsilon}$ are the following: we suppose that, for all $\varepsilon>0, \nabla_{x} \cdot \mathbf{A}^{\varepsilon}=0$ and that, for some $q>1$,

$$
\mathbf{A}^{\varepsilon} \text { 2-scale converges to } \mathcal{A} \in L^{\infty}\left(0, T ; L_{2 \pi}^{\infty}\left(\mathbb{R}_{\tau} ; W^{1, q}(K)\right)\right),
$$

for all compact sets $K \subset \mathbb{R}^{n}$ and where $\mathcal{A} \equiv \mathcal{A}(t, \tau, \mathbf{x})$ is $\theta$-periodic in $\tau$.

The proof of Theorem 1.5 begins as the proof of Theorem 1.3 in the sense that the constraint equation and its consequences are similar. Hence relation (1.28) is obvious. In order to get the equation $U_{0}$ satisfies, we proceed as follow: we define $w^{\varepsilon}(t, \mathbf{x})=u^{\varepsilon}\left(t, e^{\frac{t}{\varepsilon} M}(\mathbf{x}-\bar{N})+\bar{N}\right)$ which is the function $u^{\varepsilon}$ from which the essential oscillation is removed. This idea has also been used in $\mathrm{E}$. Frénod \& E. Sonnendrücker [7], E. Grenier [12, 13], Schochet [18], J. L. Joly, G. Metivier \& J. Rauch [15] and T. Colin [5]. Using the equation satisfied by $w^{\varepsilon}$, we show that

$$
w^{\varepsilon} \rightarrow \theta U_{0} \text { in } L^{\infty}\left(0, T ;\left(W_{0}^{1, q}(K)\right)^{*}\right),
$$

where $\left(W_{0}^{1, q}(K)\right)^{*}$ is the dual of $\left(W_{0}^{1, q}(K)\right)$. This fact coupled with the assumption on $\mathbf{A}^{\varepsilon}$ enables to pass to the limit and find equation (1.29).

Under assumption (1.26) we may deduce, up to subsequences,

$$
u^{\varepsilon} 2 \text {-scale converges to } U \in L^{\infty}\left(0, T ; L_{\theta}^{\infty}\left(\mathbb{R}_{\tau} ; L^{p}(\mathcal{O})\right)\right) .
$$

The weak formulation of (4.1) with $\theta$-periodic oscillating functions $(\psi)^{\varepsilon} \equiv \psi\left(t, \frac{t}{\varepsilon}, \mathbf{x}\right)$ writes

$$
\int_{\mathcal{Q}} u^{\varepsilon}\left(\left(\frac{\partial \psi}{\partial t}\right)^{\varepsilon}+\frac{1}{\varepsilon}\left(\frac{\partial \psi}{\partial \tau}\right)^{\varepsilon}+\mathbf{A}^{\varepsilon} \cdot\left(\nabla_{x} \psi\right)^{\varepsilon}+\frac{1}{\varepsilon} \mathbf{L} \cdot\left(\nabla_{x} \psi\right)^{\varepsilon}\right) d t d \mathbf{x}=-\int_{\mathcal{O}} u_{0} \psi(0,0, \mathbf{x}) d \mathbf{x} .
$$

Proceeding as in subsection 3.1 we obtain that $U$ satisfies

$$
\frac{\partial U}{\partial \tau}+\mathbf{L} \cdot \nabla_{x} U=0 \text { in } \mathcal{D}^{\prime}\left(\mathbb{R}_{\tau} \times \mathcal{O}\right) .
$$

and then,

$$
U(t, \tau, \mathbf{x})=U_{0}\left(t, e^{-\tau M}(\mathbf{x}-\bar{N})+\bar{N}\right),
$$

for $U_{0} \equiv U_{0}(t, \mathbf{y}) \in L^{\infty}\left(0, T ; L^{2}\left(\mathcal{O}^{\prime}\right)\right)$, which is the equation (1.28) of Theorem 1.5.

Now we look for the equation $U_{0}$ satisfies. For this purpose, we define

$$
w^{\varepsilon}(t, \mathbf{y})=u^{\varepsilon}\left(t, e^{\frac{t}{\varepsilon} M}(\mathbf{y}-\bar{N})+\bar{N}\right),
$$

and we have the following Lemma characterizing the asymptotic limit of $w^{\varepsilon}$. 
Lemma 4.1 The sequence $\left(w^{\varepsilon}\right)$ satisfies

$$
w^{\varepsilon} \rightarrow \theta U_{0} \text { in } L^{\infty}\left(0, T ;\left(W_{0}^{1, q}(K)\right)^{*}\right)
$$

where $U_{0}$ in linked with the profile $U$ by (1.28).

Proof. First, we prove that $w^{\varepsilon} 2$-scale converges to $U_{0}$, and $w^{\varepsilon}$ weakly-* converges to $\theta U_{0}$. Secondly, we show that $\frac{\partial w^{\varepsilon}}{\partial t}$ is bounded in $L^{\infty}\left(0, T ;\left(W_{0}^{1, r}(K)\right)^{*}\right)$ for some $r>1$. Since $w^{\varepsilon}$ is bounded in $L^{\infty}\left(0, T ; L^{p}(\mathcal{O})\right)$, the Aubin-Lions Lemma leads to the conclusion.

We take any function $\phi(t, \tau, \mathbf{y})$ regular, with compact support in $t$ and $\mathbf{y}$ and $\theta$-periodic with respect to $\tau$. We have:

$$
\begin{aligned}
\int_{\mathcal{O}^{\prime}} w^{\varepsilon}(t, \mathbf{y}) \phi\left(t, \frac{t}{\varepsilon}, \mathbf{y}\right) d t d \mathbf{y}=\int_{\mathcal{O}^{\prime}} u^{\varepsilon}\left(t, e^{\frac{t}{\varepsilon} M}(\mathbf{y}-\bar{N})+\bar{N}\right) \phi\left(t, \frac{t}{\varepsilon}, \mathbf{y}\right) d t d \mathbf{y}= \\
\int_{\mathcal{O}} u^{\varepsilon}(t, \mathbf{x}) \phi\left(t, \frac{t}{\varepsilon}, e^{-\frac{t}{\varepsilon} M}(\mathbf{x}-\bar{N})+\bar{N}\right) d t d \mathbf{x} .
\end{aligned}
$$

This last quantity converges to

$$
\int_{\mathcal{O}} \int_{0}^{\theta} U(t, \tau, \mathbf{x}) \phi\left(t, \tau, e^{-\tau M}(\mathbf{x}-\bar{N})+\bar{N}\right) d t d \mathbf{x} d \tau=\int_{\mathcal{O}} \int_{0}^{\theta} U_{0} \phi d t d \mathbf{y} d \tau
$$

proving $w^{\varepsilon} 2$-scale converges to $U_{0}$. Since $U_{0}$ does not depend on $\tau$, we immediately deduce $w^{\varepsilon} \rightarrow \theta U_{0}$ weakly $-*$.

Now, we seek the equation $w^{\varepsilon}$ satisfies. We have

$$
\frac{\partial w^{\varepsilon}}{\partial t}(t, \mathbf{y})=\frac{\partial u^{\varepsilon}}{\partial t}\left(t, e^{\frac{t}{\varepsilon} M}(\mathbf{y}-\bar{N})+\bar{N}\right)+\frac{M}{\varepsilon} e^{\frac{t}{\varepsilon} M}(\mathbf{y}-\bar{N}) \cdot \nabla_{x} u^{\varepsilon}\left(t, e^{\frac{t}{\varepsilon} M}(\mathbf{y}-\bar{N})+\bar{N}\right) ;
$$

writing this last equality in $\mathbf{y}=e^{-\frac{t}{\varepsilon} M}(\mathbf{x}-\bar{N})+\bar{N}$ we obtain

$$
\frac{\partial w^{\varepsilon}}{\partial t}\left(t, e^{-\frac{t}{\varepsilon} M}(\mathbf{x}-\bar{N})+\bar{N}\right)=\frac{\partial u^{\varepsilon}}{\partial t}(t, \mathbf{x})+\frac{M}{\varepsilon}(\mathbf{x}-\bar{N}) \cdot \nabla_{x} u^{\varepsilon}(t, \mathbf{x})=\frac{\partial u^{\varepsilon}}{\partial t}(t, \mathbf{x})+\frac{1}{\varepsilon} \mathbf{L} \cdot \nabla_{x} u^{\varepsilon}(t, \mathbf{x})
$$

Hence in view of the equation satisfied by $u^{\varepsilon}$ and of

$$
\nabla_{y} w^{\varepsilon}(t, \mathbf{y})=\left(e^{\frac{t}{\varepsilon} M}\right)^{T} \nabla_{x} u^{\varepsilon}\left(t, e^{\frac{t}{\varepsilon} M}(\mathbf{y}-\bar{N})+\bar{N}\right),
$$

we obtain that $w^{\varepsilon}$ is solution of

$$
\frac{\partial w^{\varepsilon}}{\partial t}+\mathbf{A}^{\varepsilon}\left(t, e^{\frac{t}{\varepsilon} M}(\mathbf{y}-\bar{N})+\bar{N}\right) \cdot\left(e^{-\frac{t}{\varepsilon} M}\right)^{T} \nabla_{y} w^{\varepsilon}=0
$$

i.e.

$$
\frac{\partial w^{\varepsilon}}{\partial t}+e^{-\frac{t}{\varepsilon} M} \mathbf{A}^{\varepsilon}\left(t, e^{\frac{t}{\varepsilon} M}(\mathbf{y}-\bar{N})+\bar{N}\right) \cdot \nabla_{y} w^{\varepsilon}=0
$$

Having (4.16) at hand we can prove that $\frac{\partial w^{\varepsilon}}{\partial t}$ is bounded in $L^{\infty}\left(0, T ;\left(W_{0}^{1, r}(K)\right)^{*}\right)$ for some $r>1$ and any compact $K \subset \mathbb{R}^{n}$. It is an easy game to show

$$
\nabla_{y} \cdot\left[e^{-\frac{t}{\varepsilon} M} \mathbf{A}^{\varepsilon}\left(t, e^{\frac{t}{\varepsilon} M}(\mathbf{y}-\bar{N})+\bar{N}\right)\right]=0 .
$$

$\mathrm{RR} \mathrm{n}^{\circ} 3847$ 
Hence, from (4.16) we deduce

$$
\frac{\partial w^{\varepsilon}}{\partial t}=-\nabla_{y} \cdot\left[e^{-\frac{t}{\varepsilon} M} \mathbf{A}^{\varepsilon}\left(t, e^{\frac{t}{\varepsilon} M}(\mathbf{y}-\bar{N})+\bar{N}\right) w^{\varepsilon}\right],
$$

and since $\mathbf{A}^{\varepsilon}$ is bounded in $W_{0}^{1, q}(K)$, a Sobolev embedding theorem implies that $\mathbf{A}^{\varepsilon}$ is bounded in $L^{q^{\prime}}(K)$, where $q^{\prime}$ is defined by $\frac{1}{q}^{\prime}=\operatorname{Max}\left(\frac{1}{q}-\frac{1}{n}, 0\right)$, and since $\tau \mapsto e^{-\tau M}$ is periodic, we deduce that $\left(e^{-\frac{t}{\varepsilon} M} \mathbf{A}^{\varepsilon}\left(t, e^{\frac{t}{\varepsilon} M}(\mathbf{y}-\bar{N})+\bar{N}\right)\right)$ is also bounded in $L^{q^{\prime}}(K)$. Then as $w^{\varepsilon}$ is bounded in $L^{p}(\mathcal{O})$, we get that $\left(e^{-\frac{t}{\varepsilon} M} \mathbf{A}^{\varepsilon}\left(t, e^{\frac{t}{\varepsilon} M}(\mathbf{y}-\bar{N})+\bar{N}\right) w^{\varepsilon}\right)$ is bounded in $L^{r^{*}}(K)$ with $\frac{1}{r^{*}}=\frac{1}{p}+\frac{1}{q^{\prime}}$. We may then conclude that

$$
\frac{\partial w^{\varepsilon}}{\partial t} \text { is bounded in } L^{\infty}\left(0, T ;\left(W_{0}^{1, r}(K)\right)^{*}\right) \text {, with } \frac{1}{r^{*}}+\frac{1}{r}=1
$$

for any compact $K \subset \mathbb{R}^{n}$.

In order to conclude, we treat first the case when $r^{*}<q^{*}$ where $q^{*}$ is such that $\frac{1}{q^{*}}+\frac{1}{q}=1$. As $K$ is compact, we have $L^{q^{*}}(K) \subset L^{r^{*}}(K)$. Since $\left(W_{0}^{1, q}(K)\right)^{*}$ and $\left(W_{0}^{1, r}(K)\right)^{*}$ are respectively isomorphic to $\left(L^{q^{*}}(K)\right)^{2}$ and $\left(L^{r^{*}}(K)\right)^{2}$ we deduce, on the one hand,

$$
\left(W_{0}^{1, q}(K)\right)^{*} \subset\left(W_{0}^{1, r}(K)\right)^{*}
$$

with continuous injection. On the other hand, as a consequence of the Rellich-Kondrachov Theorem, we have

$$
L^{p}(K) \subset\left(W_{0}^{1, q}(K)\right)^{*} \text { compactly. }
$$

Hence, applying the Aubin-Lions Lemma, (see for example Lions [16]) we deduce

$$
\mathcal{U}=\left\{u \in L^{\infty}\left(0, T ; L^{p}(K)\right), \frac{\partial u}{\partial t} \in L^{\infty}\left(0, T ;\left(W_{0}^{1, r}(K)\right)^{*}\right)\right\}
$$

is compactly embedded in $L^{\infty}\left(0, T ;\left(W_{0}^{1, q}(K)\right)^{*}\right)$. Then we deduce that $w^{\varepsilon}$ converges strongly in $L^{\infty}\left(0, T ;\left(W_{0}^{1, r}(K)\right)^{*}\right)$ giving the conclusion of the Lemma.

The case $r^{*}>q^{*}$ is simpler. Indeed, in this case, we directly have from (4.19)

$$
\frac{\partial w^{\varepsilon}}{\partial t} \text { is bounded in } L^{\infty}\left(0, T ;\left(W_{0}^{1, q}(K)\right)^{*}\right),
$$

yielding directly the conclusion of the Lemma.

Having Lemma 4.1 at hand, we want to pass to the limit in (4.16). This will give the equation for $U_{0}$. In order to realize this, we have first to prove:

LEMma 4.2 We have

$$
\begin{aligned}
& \mathbf{A}^{\varepsilon}\left(t, e^{\frac{t}{\varepsilon} M}(\mathbf{y}-\bar{N})+\bar{N}\right) \text { 2-scale converges to } \mathcal{A}\left(t, \tau, e^{\tau M}(\mathbf{y}-\bar{N})+\bar{N}\right) \\
& \qquad \text { in } L^{\infty}\left(0, T ; L_{\theta}^{\infty}\left(\mathbb{R}_{\tau} ; W_{0}^{1, q}(K)\right)\right) .
\end{aligned}
$$

Proof. A direct computation gives

$$
\begin{aligned}
& \int_{\mathcal{Q}^{\prime}} \mathbf{A}^{\varepsilon}\left(t, e^{\frac{t}{\varepsilon} M}(\mathbf{y}-\bar{N})+\bar{N}\right) \psi\left(t, \frac{t}{\varepsilon}, \mathbf{y}\right) d t d \mathbf{y}= \\
& \left.\left.\int_{\mathcal{Q}} \mathbf{A}^{\varepsilon}(t, \mathbf{x}) \psi\left(t, \frac{t}{\varepsilon}, e^{-\frac{t}{\varepsilon} M}(\mathbf{x}-\bar{N})+\bar{N}\right)\right) d t d \mathbf{x} \rightarrow \int_{\mathcal{Q}} \int_{0}^{\theta} \mathcal{A}(t, \tau, \mathbf{x}) \psi\left(t, \tau, e^{-\tau M}(\mathbf{x}-\bar{N})+\bar{N}\right)\right) d t d \mathbf{y} d \tau \\
& \left.\quad=\int_{\mathcal{Q}^{\prime}} \int_{0}^{\theta} \mathcal{A}\left(t, \tau, e^{\tau M}(\mathbf{y}-\bar{N})+\bar{N}\right)\right) \psi(t, \tau, \mathbf{y}) d t d \mathbf{y} d \tau,
\end{aligned}
$$


for any $\theta$-periodic test function. This proves the Lemma.

Now, writing a weak formulation of (4.16), we have

$$
\int_{\mathcal{Q}^{\prime}} w^{\varepsilon}\left[\frac{\partial \varphi}{\partial t}+e^{-\frac{t}{\varepsilon} M} \mathbf{A}^{\varepsilon}\left(t, e^{\frac{t}{\varepsilon} M}(\mathbf{y}-\bar{N})+\bar{N}\right) \cdot \nabla_{y} \varphi\right] d t d \mathbf{y}=\int_{\mathcal{O}^{\prime}} u_{0} \varphi(0, \cdot) d \mathbf{y}
$$

for any $\varphi(t, \mathbf{y})$ regular and compactly supported in $\mathcal{Q}$. Let $K$ be a compact containing the support of $\varphi$, since

$$
\begin{aligned}
& w^{\varepsilon} \rightarrow \theta U_{0} \text { in } L^{\infty}\left(0, T ;\left(W_{0}^{1, q}(K)\right)^{*}\right), \\
& \mathbf{A}^{\varepsilon}\left(t, e^{\frac{t}{\varepsilon} M}(\mathbf{y}-\bar{N})+\bar{N}\right) 2 \text {-scale converges to } \mathcal{A}\left(t, e^{\tau M}(\mathbf{y}-\bar{N})+\bar{N}\right) \\
& \text { in } L^{\infty}\left(0, T ; L_{\theta}^{\infty}\left(\mathbb{R}_{\tau} ; W_{0}^{1, q}(K)\right)\right),
\end{aligned}
$$

we can pass to the limit in (4.26) and find:

$$
\int_{\mathcal{Q}^{\prime}} \theta U_{0}\left[\frac{\partial \varphi}{\partial t}+\int_{0}^{\theta} e^{-\tau M} \mathcal{A}\left(t, \tau, e^{\tau M}(\mathbf{y}-\bar{N})+\bar{N}\right) d \tau \cdot \nabla_{y} \varphi\right] d t d \mathbf{y}=\int_{\mathcal{O}^{\prime}} u_{0} \varphi(0, \cdot) d \mathbf{y} .
$$

Noticing at last that neither $U_{0}$ nor $\varphi$ depend on $\tau,(4.28)$ is nothing but a weak formulation of (1.29) proving Theorem 1.5.

Remark 4.1 In the case when $\mathbf{A}$ does not depend on $\varepsilon$, its 2 -scale limit is $\frac{\mathbf{A}}{\theta}$, so that we indeed get the same result as in Theorem 1.3.

\subsection{Application to the 2D Vlasov-Poisson system - Proof of Theorem 1.4}

In order to deduce Theorem 1.4 from Theorem 1.5 we essentially have to show (1.20) and to pass to the limit in the Poisson equation. Indeed, once those two things are proved, the Theorem follows noticing that the Vlasov equation (1.17.a) enters the generalized generic framework with

$$
\mathbf{A}^{\varepsilon}(t, \mathbf{x}, \mathbf{v})=\left(\begin{array}{c}
0 \\
\mathbf{E}^{\varepsilon}(t, \mathbf{x})
\end{array}\right)\left(\in \mathbb{R}^{4}\right) \text { and } \mathbf{L}(t, \mathbf{x}, \mathbf{v})=\left(\begin{array}{c}
\mathbf{v} \\
\mathbf{v} \times \mathcal{M}
\end{array}\right)\left(\in \mathbb{R}^{4}\right)
$$

In this case $e^{\tau M}$ becomes

$$
e^{\tau M}=\left(\begin{array}{ll}
I & \mathcal{R}(\tau) \\
0 & R(\tau)
\end{array}\right)
$$

with $\mathcal{R}(\tau)$ and $R(\tau)$ given by (1.23).

Multiplying the Vlasov equation (1.17.a) by $\left(f^{\varepsilon}\right)^{p-1}$ and integrating in $\mathbf{x}$ and $\mathbf{v}$ we obtain

$$
\left\|f^{\varepsilon}\right\|_{L^{\infty}\left(0, T ; L^{p}(\mathcal{O})\right)} \leq C
$$

for some constants $C$. From this estimate, we deduce

Lemma 4.3 Under assumption (1.19)

$$
f^{\varepsilon} \text { 2-scale converges to } F \in L^{\infty}\left(0, T ; L_{2 \pi}^{\infty}\left(\mathbb{R}_{\tau} ; L^{p}(\mathcal{O})\right)\right),
$$

$\mathrm{RR} \mathrm{n}^{\circ} 3847$ 
The fact that $\mathbf{E}^{\varepsilon} 2$-scale converges is a bit longer to obtain. We need first to show the following Lemma.

Lemma 4.4 Under assumption (1.19), we have

$$
\left\|\left(|v|^{2} f^{\varepsilon}\right)\right\|_{L^{\infty}\left(0, T ; L^{1}(\mathcal{O})\right)} \leq C \text { and }\left\|\rho^{\varepsilon}(\mathbf{x}, t)\right\|_{L^{\infty}\left(0, T ; L^{\frac{3}{2}}\left(\mathbb{R}_{x}^{3}\right)\right)} \leq C,
$$

for some constant $C$.

Proof. Multiplying the Vlasov equation by $|\mathbf{v}|^{2}$, and integrating with respect to $\mathbf{x}$ and $\mathbf{v}$, we get

$$
\frac{d}{d t} \int_{\mathcal{O}} f^{\varepsilon}|\mathbf{v}|^{2} d \mathbf{v} d \mathbf{x}-2 \int_{\mathbb{R}_{x}^{2}} \mathbf{J}^{\varepsilon} \cdot \mathbf{E}^{\varepsilon} d \mathbf{x}=0
$$

where

$$
\mathbf{J}^{\varepsilon}(\mathbf{x}, t)=\int_{\mathbb{R}_{v}^{2}} \mathbf{v} f^{\varepsilon} d \mathbf{v}
$$

Now, integrating the Vlasov equation in $\mathbf{v}$ gives the continuity equation

$$
\frac{\partial \rho^{\varepsilon}}{\partial t}+\frac{1}{\varepsilon} \nabla \cdot \mathbf{J}^{\varepsilon}=0
$$

Using this, we obtain

$$
\int_{\mathbb{R}_{\boldsymbol{x}}^{2}} \mathbf{J}^{\varepsilon} \cdot \mathbf{E}^{\varepsilon} d \mathbf{x}=-\int_{\mathbb{R}_{\boldsymbol{x}}^{2}} \mathbf{J}^{\varepsilon} \cdot \nabla \phi^{\varepsilon} d \mathbf{x}=\int_{\mathbb{R}_{\boldsymbol{x}}^{2}} \nabla \cdot \mathbf{J}^{\varepsilon} \phi^{\varepsilon} d \mathbf{x}=-\varepsilon \int_{\mathbb{R}_{\boldsymbol{x}}^{2}} \frac{\partial \rho_{\varepsilon}}{\partial t} \phi^{\varepsilon} d \mathbf{x} .
$$

Using now the Poisson equation, we get

$$
\frac{1}{2} \frac{d}{d t} \int_{\mathbb{R}_{x}^{2}}\left(\nabla \phi^{\varepsilon}\right)^{2} d \mathbf{x}=-\int_{\mathbb{R}_{x}^{2}} \frac{\partial}{\partial t} \Delta \phi^{\varepsilon} \phi^{\varepsilon} d \mathbf{x}=\int_{\mathbb{R}_{x}^{2}} \frac{\partial \rho_{\varepsilon}}{\partial t} \phi^{\varepsilon} d \mathbf{x} .
$$

Coupling (4.37) and (4.38) yields:

$$
-2 \int_{\mathbb{R}_{x}^{2}} \mathbf{J}^{\varepsilon} \cdot \mathbf{E}^{\varepsilon} d \mathbf{x}=\varepsilon \frac{d}{d t} \int_{\mathbb{R}_{x}^{2}}\left(\nabla \phi^{\varepsilon}\right)^{2} d \mathbf{x}
$$

and then, (4.34) reads

$$
\frac{d}{d t}\left[\int_{\mathcal{O}} f^{\varepsilon}|\mathbf{v}|^{2} d \mathbf{v} d \mathbf{x}+\varepsilon \int_{\mathbb{R}_{x}^{2}}\left(\nabla \phi^{\varepsilon}\right)^{2} d \mathbf{x},\right]=0
$$

and as an immediate consequence we have

$$
\left\|\left(|v|^{2} f^{\varepsilon}\right)\right\|_{L^{\infty}\left(0, T ; L^{1}(\mathcal{O})\right)} \leq C,
$$

for some constant $C$. The first part of the Lemma is then proved.

Concerning $\rho^{\varepsilon}$ we have

$$
\rho^{\varepsilon}(\mathbf{x}, t)=\int_{\mathbb{R}_{v}^{2}} f^{\varepsilon} d \mathbf{v}=\int_{|v|<R} f^{\varepsilon} d \mathbf{v}+\int_{|v|>R} f^{\varepsilon} d \mathbf{v}
$$


for any $R>0$. Using the Cauchy-Schwartz inequality, we have

$$
\int_{|v|<R} f^{\varepsilon} d \mathbf{v} \leq\left(\int_{|v|<R}\left(f^{\varepsilon}\right)^{2} d \mathbf{v}\right)^{\frac{1}{2}}\left(\int_{|v|<R} d \mathbf{v}\right)^{\frac{1}{2}} \leq C_{1} R\left(\int_{\mathbb{R}_{v}^{2}}\left(f^{\varepsilon}\right)^{2} d \mathbf{v}\right)^{\frac{1}{2}},
$$

and

$$
\int_{|v|>R} f^{\varepsilon} d \mathbf{v} \leq \int_{|v|>R} \frac{|v|^{2}}{R^{2}} f^{\varepsilon} d \mathbf{v} \leq \frac{1}{R^{2}} \int_{\mathbb{R}_{v}^{2}}|v|^{2} f^{\varepsilon} d \mathbf{v} .
$$

Hence, we have for any $R>0$

$$
\left|\rho^{\varepsilon}(\mathbf{x}, t)\right| \leq C_{1} R\left(\int_{\mathbb{R}_{v}^{2}}\left(f^{\varepsilon}\right)^{2} d \mathbf{v}\right)^{\frac{1}{2}}+\frac{1}{R^{2}} \int_{\mathbb{R}_{v}^{2}}|v|^{2} f^{\varepsilon} d \mathbf{v} .
$$

Taking the $R$ which minimizes the right-hand-side we obtain

$$
\left|\rho^{\varepsilon}(\mathbf{x}, t)\right| \leq C_{2}\left(\int_{\mathbb{R}_{v}^{2}}\left(f^{\varepsilon}\right)^{2} d \mathbf{v}\right)^{\frac{1}{3}}\left(\int_{\mathbb{R}_{v}^{2}}|v|^{2} f^{\varepsilon} d \mathbf{v}\right)^{\frac{1}{3}}
$$

and finally

$$
\begin{aligned}
\int_{\mathbb{R}_{x}^{2}}\left|\rho^{\varepsilon}(\mathbf{x}, t)\right|^{\frac{3}{2}} d \mathbf{x} & \leq C_{3} \int_{\mathbb{R}_{x}^{2}}\left(\int_{\mathbb{R}_{v}^{2}}\left(f^{\varepsilon}\right)^{2} d \mathbf{v}\right)^{\frac{1}{2}}\left(\int_{\mathbb{R}_{v}^{2}}|v|^{2} f^{\varepsilon} d \mathbf{v}\right)^{\frac{1}{2}} d \mathbf{x} \\
& \leq C_{3}\left(\int_{\mathbb{R}_{x}^{2} \times \mathbb{R}_{v}^{2}}\left(f^{\varepsilon}\right)^{2} d \mathbf{x} d \mathbf{v}\right)^{\frac{1}{2}}\left(\int_{\mathbb{R}_{x}^{2} \times \mathbb{R}_{v}^{2}}|v|^{2} f^{\varepsilon} d \mathbf{x} d \mathbf{v}\right)^{\frac{1}{2}}
\end{aligned}
$$

thanks to the Hölder inequality. Now, knowing that the terms on the right-hand-side are bounded, we have our estimate on $\rho^{\varepsilon}$. Hence the proof of the Lemma is ended.

As a direct consequence of Lemma 4.4, and of the regularization properties of the Laplace operator, we deduce that $\mathbf{E}^{\varepsilon}$ is bounded in $L^{\infty}\left(0, T ; W^{1, \frac{3}{2}}\left(\mathbb{R}_{x}^{2}\right)\right)$ and the following Lemma holds true

Lemma 4.5 Extracting a subsequence, we have

$$
E^{\varepsilon} \text { 2-scale converges to } \mathcal{E} \in L^{\infty}\left(0, T ; L_{2 \pi}^{\infty}\left(\mathbb{R}_{\tau} ; W^{1, \frac{3}{2}}\left(\mathbb{R}_{x}^{2}\right)\right)\right)
$$

Hence we proved the two facts yielding (1.22.a) and (1.22.b) with the help of Theorem 1.5.

It now remains to pass to the 2 -scale limit in the Poisson equation (1.17). This can be done easily writing a weak formulation of the Poisson equation with oscillating test functions:

$$
\int_{\mathbb{R}_{x}^{2}} \nabla \phi^{\varepsilon}(t, \mathbf{x}) \cdot \nabla \psi\left(t, \frac{t}{\varepsilon}, \mathbf{x}\right) d t d \mathbf{x}=\int_{\mathcal{O}} f^{\varepsilon}(t, \mathbf{x}, \mathbf{v}) \psi\left(t, \frac{t}{\varepsilon}, \mathbf{x}\right) d t d \mathbf{x} d \mathbf{v}
$$

in which we can pass to the limit and obtain, denoting $\Phi$ the 2-scale limit of $\phi^{\varepsilon}$

$$
\begin{aligned}
\int_{\mathbb{R}_{x}^{2}} \int_{0}^{2 \pi} \nabla \Phi \cdot \nabla \psi d t d \mathbf{x} d \tau & =\int_{\mathcal{O}} \int_{0}^{2 \pi} F \psi d t d \mathbf{x} d \mathbf{v} d \tau \\
& =\int_{\mathcal{O}} \int_{0}^{2 \pi} G(t, \mathbf{x}+\mathcal{R}(-\tau) \mathbf{v}, R(-\tau) \mathbf{v}) \psi d t d \mathbf{x} d \mathbf{v} d \tau
\end{aligned}
$$

which is the weak formulation of (1.22.c), achieving, in view of what is said in the beginning of the subsection, the proof of Theorem 1.4,.

$\mathrm{RR} \mathrm{n}^{\circ} 3847$ 
REMARK 4.2 The deduction of the equation satisfied by the weak-* limit $f$ from (1.22) is an open problem. Indeed, writing an equation for $[G(t, \mathbf{x}+\mathcal{R}(-\tau) \mathbf{v}, R(-\tau) \mathbf{v})]$ from (1.22) introduce the $\tau$-variable in the coefficients of $\nabla_{x}[G(t, \mathbf{x}+\mathcal{R}(-\tau) \mathbf{v}, R(-\tau) \mathbf{v})]$ and $\nabla_{v}[G(t, \mathbf{x}+\mathcal{R}(-\tau) \mathbf{v}, R(-\tau) \mathbf{v})]$. Hence we cannot proceed as in the linear case. Moreover, since those coefficients also depend on $\mathbf{x}$ and $\mathbf{v}$, the non local homogenization methods (see L. Tartar [19, 20], Y. Amirat, K. Hamdache \& A. Ziani [3, 4] E. Frenod \& K. Hamdache [6], R. Alexandre [1] ... ) do not work.

\section{References}

[1] Alexandre, R. Some results in homogenization tackling memory effects.

[2] Allaire, G. Homogeneization and Two-scale Convergence. SIAM J. Math. Anal. XXIII, 6 (1992), 1482-1518.

[3] Amirat, Y., Hamdache, K., and Ziani, A. Homogénéisation d'équations hyperboliques $\mathrm{du}$ premier ordre et application aux écoulements missibles en milieux poreux. Ann. Inst. H. Poincaré 6, 5 (1989), 397-417.

[4] Amirat, Y., Hamdache, K., and Ziani, A. Homogenisation of parametrised families of hyperbolic problems. Proc. Royal Soc. Edinburgh 120A (1992), 199-221.

[5] Colin, T. Rigorous derivation of the non linear schrödinger equation and davey-stewartson system from quadratic hyperbolic systems. Personnal communication.

[6] Frénod, E., and Hamdache, K. Homogeneization of kinetic equations with oscillating potentials. Proc. Royal Soc. Edinburgh 126A (1996), 1247-1275.

[7] Frénod, E., And Sonnendrücker, E. Long time behavior of the Vlasov equation with a strong external magnetic field. INRIA report 3428 (1998).

[8] Frénod, E., and Sonnendrücker, E. Approximation rayon de larmor fini de l'équation de Vlasov. Note soumise (1999).

[9] Frénod, E., And Sonnendrücker, E. Homogenization of the Vlasov equation and of the Vlasov-Poisson system with a strong external magnetic field. Asymptotic Analysis (accepted).

[10] Golse, F., and Saint Raymond, L. L'approximation centre guide pour l'équation de vlasovpoisson. C. R. Acad. Sci. Paris, Série I 328, 10 (1998), 865-870.

[11] Golse, F., and Saint Raymond, L. The vlasov-poisson system with strong magnetic field. J. Math. Pures. Appl. (1999).

[12] Grenier, E. Oscillatory perturbation of the navier-stokes equations. J. Maths. Pures Appl. 76 (1997), 477-498.

[13] Grenier, E. Pseudo-differential energy estimates of singular perturbations. Comm. Pure Appl. Maths. 50 (1997), 821-865.

[14] Hansen, F., Knorr, G., Lynov, J., Pécseli, H., and Juul Rasmussen, J. A numerical plasma simulation including finite larmor radius effects to arbitrary order. Plasma Physics and Controlled Fusion 31, 2 (1989), 173-183.

[15] Joly, J. L., MÉtivier, G., and Rauch, J. 
[16] Lions, J. L. Quelques méthodes de résolution de problèmes aux limites non linéaires. Dunod, Gauthier-Villars, 1969.

[17] N'Guetseng, G. A general convergence result for a functional related to the theory of homogenization. SIAM J. Math. Anal. 20, 3 (1989), 608-623.

[18] Schochet, S. Fast singular limit of hyperbolic pdes. J. Diff. Equ. 114 (1994), 476-512.

[19] Tartar, L. Non local effects induced by homogenization. Essays of Mathematical analysis in Honour of E. De Giorgi (Birkhäuser, Boston) (1989).

[20] Tartar, L. Memory effects an homogenization. Arch. Rat. Mech. Anal. (1990), 121-133. 
Unité de recherche INRIA Lorraine, Technopôle de Nancy-Brabois, Campus scientifique, 615 rue du Jardin Botanique, BP 101, 54600 VILLERS LÈS NANCY

Unité de recherche INRIA Rennes, Irisa, Campus universitaire de Beaulieu, 35042 RENNES Cedex Unité de recherche INRIA Rhône-Alpes, 655, avenue de l'Europe, 38330 MONTBONNOT ST MARTIN Unité de recherche INRIA Rocquencourt, Domaine de Voluceau, Rocquencourt, BP 105, 78153 LE CHESNAY Cedex Unité de recherche INRIA Sophia-Antipolis, 2004 route des Lucioles, BP 93, 06902 SOPHIA-ANTIPOLIS Cedex

Éditeur

INRIA, Domaine de Voluceau, Rocquencourt, BP 105, 78153 LE CHESNAY Cedex (France)

http://www.inria.fr

ISSN 0249-6399 